\title{
Solutions of associative Yang-Baxter equation and $D$-equation in low dimensions and associated Frobenius algebras and Connes cocycles
}

\author{
Mahouton Norbert Hounkonnou* and Gbêvèwou Damien Houndedji ${ }^{\dagger}$
}

November 13, 2018

\begin{abstract}
This work addresses some relevant characteristics of associative algebras in low dimensions. Especially, given 1 and 2 dimensional associative algebras, we explicitly solve associative Yang-Baxter equations and use skew-symmetric solutions to perform double constructions of Frobenius algebras. Besides, we determine related compatible dendriform algebras and solutions of their $D$-equations. Finally, using symmetric solutions of the latter equations, we proceed to double constructions of corresponding Connes cocycles.

Keywords. Associative algebra, Frobenius algebra, Connes cocycle, dendriform algebra, Dequation, Yang-Baxter equation.
\end{abstract}

MSC2010. 16T25, 05C25, 16S99, 16Z05.

\section{Introduction}

A (symmetric) Frobenius algebra which is an associative algebra with a (symmetric) nondegenerate invariant bilinear form is an important object in both mathematics and mathematical physics. It plays a key role in the study of many topics, such as statistical models over 2-dimensional graphs [7] and topological quantum field theory [19]. On the other hand, a nondegenerate Connes cocycle is an associative algebra with a non-degenerate antisymmetric bilinear form being a cyclic 1-cocyle in the sense of Connes [11. It corresponds to the original definition of cyclic cohomology by Connes and hence is important in the study of noncommutative geometry.

However, it is not easy to construct Frobenius algebras or non-degenerate Connes cocycles explicitly, that is, both the explicit examples of Frobenius algebras and non-degenerate Connes cocycles are lacked. In [5], some special constructions (namely, double constructions) of both two objects were given in terms of bialgebra structures and certain algebraic equations. In particular, it provides an approach to construct both (symmetric) Frobenius algebras and non-degenerate Connes cocycles from solving certain algebraic equations. Explicitly, a (symmetric) Frobenius algebra can be obtained from an anti-symmetric solution of associative Yang-Baxter equation in an associative algebra, whereas a non-degenerate Connes cocycle can be obtained from a symmetric solution of $D$-equation in a dendriform algebra which is the underlying algebraic structure of a non-degenerate Connes cocycle. Note that both associative Yang-Baxter equation and dendriform algebras appeared more early in some other fields. For example, the associative Yang-Baxter equation was introduced by Aguiar 1 ] to study the cases of principal derivations for the infinitesimal bialgebras given by Joni and Rota $\mathbf{1 8}$ to provide an algebraic framework for the calculus of divided difference, whereas dendriform algebras were introduced by Loday [20] with motivation from algebraic K-theory and were studied quite extensively with connections to several areas in mathematics and physics, like operads [22], homology [14], [15], arithmetics [21] and quantum field theory $[\mathbf{1 3}$.

Preprint: ICMPA-MPA/2015/08 
In this paper, under the above framework, we will give the explicit study in low dimensions. The paper is organized as follows. In Section 2, we give some basic notions and results on the double constructions of Frobenius algebras and Connes cocycles. In Section 3, we give the explicit study in dimension 1. Specifically, given 1-dimensional associative algebras, we explicitly solve associative Yang-Baxter equations and use skew-symmetric solutions to perform double constructions of Frobenius algebras. Then, we determine related compatible dendriform algebras and solutions of their $D$-equations. Finally, using symmetric solutions of the latter equations, we proceed to double constructions of corresponding Connes cocycles. In Section 4, similar calculations are performed in dimension 2. In Section 5, we give some concluding remarks.

\section{Preliminaries}

In this section, we give a quick overview on main definitions and fundamental results essentially known from [5]. See also [1, [26]- 29, [20 and [16] and the references therein.

Definition 2.1. A bilinear form $\mathcal{B}(\cdot, \cdot)$ on an associative algebra $\mathcal{A}$ is invariant if

$$
\mathcal{B}(x y, z)=\mathcal{B}(x, y z) \text { for all } x, y, z \in \mathcal{A} \text {. }
$$

Definition 2.2. An antisymmetric bilinear form $\omega(\cdot, \cdot)$ on an associative algebra $\mathcal{A}$ is a cyclic 1-cocycle in the sense of Connes if

$$
\omega(x y, z)+\omega(y z, x)+\omega(z x, y)=0 \text { for all } x, y, z \in \mathcal{A} .
$$

For simplicity, $\omega$ is called a Connes cocycle.

Definition 2.3. A Frobenius algebra $(\mathcal{A}, \mathcal{B})$ is an associative algebra $\mathcal{A}$ with a non-degenerate invariant bilinear form $\mathcal{B}(\cdot, \cdot)$. It is symmetric if $\mathcal{B}$ is symmetric.

Definition 2.4. We call $(\mathcal{A}, \mathcal{B})$ a double construction of a (symmetric) Frobenius algebra associated to $\mathcal{A}_{1}$ and $\mathcal{A}_{1}^{*}$ if it satisfies the conditions

(1) $\mathcal{A}=\mathcal{A}_{1} \oplus \mathcal{A}_{1}^{*}$ as the direct sum of vector spaces;

(2) $\mathcal{A}_{1}$ and $\mathcal{A}_{1}^{*}$ are associative subalgebras of $\mathcal{A}$;

(3) $\mathcal{B}$ is the natural symmetric bilinear form on $\mathcal{A}_{1} \oplus \mathcal{A}_{1}^{*}$ given by

$$
\mathcal{B}\left(x+a^{*}, y+b^{*}\right)=\left\langle x, b^{*}\right\rangle+\left\langle a^{*}, y\right\rangle \text { for all } x, y \in \mathcal{A}_{1}, a^{*}, b^{*} \in \mathcal{A}_{1}^{*},
$$

where $\langle$,$\rangle is the natural pair between the vector space \mathcal{A}_{1}$ and its dual space $\mathcal{A}_{1}^{*}$.

$W$ e call $(\mathcal{A}, \omega)$ a Connes cocycle associated to $\mathcal{A}_{1}$ and $\mathcal{A}_{1}^{*}$ if it satisfies the conditions (1), (2) and

(4) $\omega$ is the natural antisymmetric bilinear form on $\mathcal{A}_{1} \oplus \mathcal{A}_{1}^{*}$ given by

$$
\omega\left(x+a^{*}, y+b^{*}\right)=-\left\langle x, b^{*}\right\rangle+\left\langle a^{*}, y\right\rangle \text { for all } x, y \in \mathcal{A}_{1}, a^{*}, b^{*} \in \mathcal{A}_{1}^{*},
$$

and $\omega$ is a Connes cocycle on $\mathcal{A}$.

Let us now give some notations useful in the sequel. Let $\mathcal{A}$ be an associative algebra.

Considering the representations of the left $L$ and right $R$ multiplication operations defined as:

$$
\begin{aligned}
L: \mathcal{A} & \longrightarrow \mathfrak{g l}(\mathcal{A}) \\
x & \longmapsto L_{x}: \mathcal{A} \longrightarrow \mathcal{A} \\
y & \longmapsto x \cdot y, \\
R: \mathcal{A} & \longrightarrow \mathfrak{g l}(\mathcal{A}) \\
x & \longmapsto R_{x}: \mathcal{A} \longrightarrow \mathcal{A} \\
y & \longmapsto y \cdot x,
\end{aligned}
$$

The dual maps $L^{*}, R^{*}$ of the linear maps $L, R$, are defined, respectively, as: $L^{*}, R^{*}: \mathcal{A} \rightarrow \mathfrak{g l}\left(\mathcal{A}^{*}\right)$ such that: 


$$
\begin{aligned}
& L^{*}: \mathcal{A} \longrightarrow \mathfrak{g l}\left(\mathcal{A}^{*}\right) \\
& \mathcal{A}^{*} \longrightarrow \mathcal{A}^{*}
\end{aligned}
$$

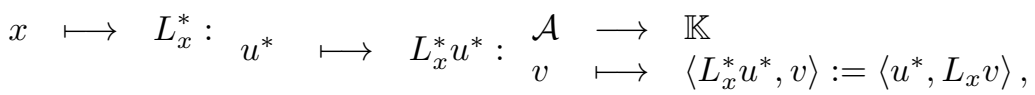

$$
\begin{aligned}
& R^{*}: \mathcal{A} \longrightarrow \mathfrak{g l}\left(\mathcal{A}^{*}\right)
\end{aligned}
$$

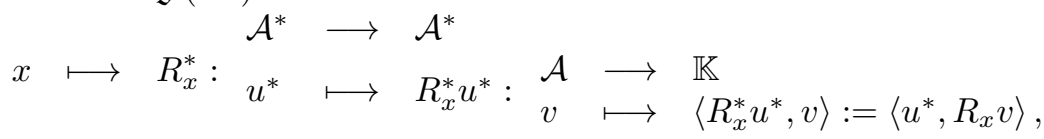

for all $x, v \in \mathcal{A}, u^{*} \in \mathcal{A}^{*}$, where $\mathcal{A}^{*}$ is the dual space of $\mathcal{A}$.

Let $\sigma: \mathcal{A} \otimes \mathcal{A} \rightarrow \mathcal{A} \otimes \mathcal{A}$ be the exchange operator defined as

$$
\sigma(x \otimes y)=y \otimes x,
$$

for all $x, y \in \mathcal{A}$.

An associative Yang-Baxter equation (AYBE) in the associative algebra $\mathcal{A}$ is defined by $\mathbf{5}$

$$
r_{12} r_{13}+r_{13} r_{23}-r_{23} r_{12}=0
$$

where $r=\sum_{i} x_{i} \otimes y_{i} \in \mathcal{A} \otimes \mathcal{A}$ and

$$
\begin{aligned}
& r_{12} r_{13}=\sum_{i, j} x_{i} x_{j} \otimes y_{i} \otimes y_{j}, \\
& r_{13} r_{23}=\sum_{i, j} x_{i} \otimes x_{j} \otimes y_{i} y_{j}, \\
& r_{23} r_{12}=\sum_{i, j} x_{j} \otimes x_{i} y_{j} \otimes y_{i} .
\end{aligned}
$$

Definition 2.5. Let $V_{1}, V_{2}$ be two vector spaces. For a linear map $\phi: V_{1} \rightarrow V_{2}$, we denote the dual (linear) map by $\phi^{*}: V_{2}^{*} \rightarrow V_{1}^{*}$ given by

$$
\left\langle v, \phi^{*}\left(u^{*}\right)\right\rangle=\left\langle\phi(v), u^{*}\right\rangle
$$

for all $v \in V_{1}, u^{*} \in V_{2}^{*}$.

DeFINITION 2.6. Let $\mathcal{A}$ be an associative algebra. An antisymmetric infinitesimal bialgebra structure on $\mathcal{A}$ is a linear map $\Delta: \mathcal{A} \rightarrow \mathcal{A} \otimes \mathcal{A}$ such that

(1) $\Delta^{*}: \mathcal{A}^{*} \otimes \mathcal{A}^{*} \rightarrow \mathcal{A}^{*}$ defines an associative algebra structure on $\mathcal{A}^{*}$;

(2) $\Delta$ satisfies the following equations:

$$
\begin{aligned}
\Delta(x \cdot y)= & (i d \otimes L(x)) \Delta(y)+(R(y) \otimes i d) \Delta(x), \\
& (L(y) \otimes i d-i d \otimes R(y)) \Delta(x) \\
& +\sigma[(L(x) \otimes i d-i d \otimes R(x)) \Delta(y)]=0,
\end{aligned}
$$

for all $x, y \in \mathcal{A}$.

We denote this bialgebra structure by $(\mathcal{A}, \Delta)$ or $\left(\mathcal{A}, \mathcal{A}^{*}\right)$.

Theorem 2.7. Let $(\mathcal{A}, \cdot)$ and $\left(\mathcal{A}^{*}, \circ\right)$ be two associative algebras. Then, the following conditions are equivalent:

(1) there is a double construction of a Frobenius algebra associated with $(\mathcal{A}, \cdot)$ and $\left(\mathcal{A}^{*}, \circ\right)$;

(2) $\left(\mathcal{A}, \mathcal{A}^{*}\right)$ is an antisymmetric infinitesimal bialgebra. 
Corollary 2.8. Let $\mathcal{A}$ be an associative algebra and $r \in \mathcal{A} \otimes \mathcal{A}$. Suppose that $r$ is antisymmetric. Then the map $\Delta$ defined by

$$
\Delta(x)=(i d \otimes L(x)-R(x) \otimes i d) r \text { for all } x \in \mathcal{A}
$$

induces an associative algebra structure on $\mathcal{A}^{*}$ such that $\left(\mathcal{A}, \mathcal{A}^{*}\right)$ is an antisymmetric infinitesimal bialgebra if (2.8) is satisfied.

Proposition 2.9. Let $(\mathcal{A}, \cdot)$ be an associative algebra and let $r \in \mathcal{A} \otimes \mathcal{A}$ be an antisymmetric solution of the associative Yang-Baxter equation in $\mathcal{A}$. Then, the corresponding double construction of Frobenius algebra $(\mathcal{A D}(\mathcal{A}), *)$ associated to $\mathcal{A}$ and $\mathcal{A}^{*}$ is given from the product in $\mathcal{A}$ as follows:

$$
\begin{gathered}
a^{*} * b^{*}=a^{*} \circ b^{*}=R^{*}\left(r\left(a^{*}\right)\right) b^{*}+L^{*}\left(r\left(b^{*}\right)\right) a^{*}, \\
x * a^{*}=x \cdot r\left(a^{*}\right)-r\left(R^{*}(x) a^{*}\right)+R^{*}(x) a^{*}, \\
a^{*} * x=r\left(a^{*}\right) \cdot x-r\left(L^{*}(x) a^{*}\right)+L^{*}(x) a^{*},
\end{gathered}
$$

for any $x \in \mathcal{A}, a^{*}, b^{*} \in \mathcal{A}^{*}$.

DeFinition 2.10. Let $\mathcal{A}$ be a vector space with two bilinear products denoted by $\prec$ and $\succ$. Then $(\mathcal{A}, \prec, \succ)$ is called a dendriform algebra if, for any $x, y, z \in \mathcal{A}$,

$$
\begin{aligned}
& (x \prec y) \prec z=x \prec(y * z), \\
& (x \succ y) \prec z=x \succ(y \prec z), \\
& x \succ(y \succ z)=(x * y) \succ z,
\end{aligned}
$$

where $x * y=x \prec y+x \succ y$.

Let $(\mathcal{A}, \prec, \succ)$ be a dendriform algebra. For any $x \in \mathcal{A}$, let $L_{\succ}(x), R_{\succ}(x)$ and $L_{\prec}(x)$, $R_{\prec}(x)$ denote the left and right multiplication operators of $(\mathcal{A}, \prec)$ and $(\mathcal{A}, \succ)$, respectively:

$$
L_{\succ}(x) y=x \succ y, R_{\succ}(x) y=y \succ x, L_{\prec}(x) y=x \prec y, R_{\prec}(x) y=y \prec x,
$$

for all $x, y \in \mathcal{A}$. Moreover, let $L_{\succ}, R_{\succ}, L_{\prec}, R_{\prec}: \mathcal{A} \rightarrow g l(\mathcal{A})$ be four linear maps with $x \mapsto$ $L_{\succ}(x), x \mapsto R_{\succ}(x), x \mapsto L_{\prec}(x)$, and $x \mapsto R_{\prec}(x)$, respectively. It is known that the product given by $[20$

$$
x * y=x \prec y+x \succ y, \text { for all } x, y \in \mathcal{A},
$$

defines an associative algebra. We call $(\mathcal{A}, *)$ the associated associative algebra of $(\mathcal{A}, \prec, \succ)$ and $(\mathcal{A}, \succ, \prec)$ is called a compatible dendriform algebra structure on the associative algebra $(\mathcal{A}, *)$.

THEOREM 2.11. Let $(\mathcal{A}, *)$ be an associative algebra and let $\omega$ be a non-degenerate Connes cocycle. Then, there exists a compatible dendriform algebra structure $\succ, \prec$ on $\mathcal{A}$ given by

$$
\omega(x \succ y, z)=\omega(y, z * x), \quad \omega(x \succ y, z)=\omega(x, y * z) \text { for all } x, y \in \mathcal{A} .
$$

Corollary 2.12. Let $\left(T(\mathcal{A})=\mathcal{A} \bowtie \mathcal{A}^{*}, \omega\right)$ be a double construction of the Connes cocycle. Then, there exists a compatible dendriform algebra structure $\succ, \prec$ on $T(\mathcal{A})$ defined by the equation (2.16). Moreover, $\mathcal{A}$ and $\mathcal{A}^{*}$, endowed with this product, are dendriform subalgebras.

Definition 2.13. Let $\mathcal{A}$ be a vector space. A dendriform D-bialgebra structure on $\mathcal{A}$ is a set of linear maps $\left(\Delta_{\prec}, \Delta_{\succ}, \beta_{\prec}, \beta_{\succ}\right)$ given by $\Delta_{\prec}, \Delta_{\succ}: \mathcal{A} \rightarrow \mathcal{A} \otimes \mathcal{A}, \quad \beta_{\prec}, \beta_{\succ}: \mathcal{A}^{*} \rightarrow \mathcal{A}^{*} \otimes \mathcal{A}^{*}$, such that

(a) $\left(\Delta_{\prec}^{*}, \Delta_{\succ}^{*}\right): \mathcal{A}^{*} \otimes \mathcal{A}^{*} \rightarrow \mathcal{A}^{*}$ defines a dendriform algebra structure $\left(\succ \mathcal{A}^{*}, \prec \mathcal{A}^{*}\right)$ on $\mathcal{A}^{*}$;

(b) $\left(\beta_{\prec}^{*}, \beta_{\succ}^{*}\right): \mathcal{A} \otimes \mathcal{A} \rightarrow A$ defines a dendriform algebra structure $\left(\succ_{\mathcal{A}}, \prec_{\mathcal{A}}\right)$ on $\mathcal{A}$;

(c) the following equations are satisfied

$$
\begin{aligned}
\Delta_{\prec}\left(x *_{\mathcal{A}} y\right) & =\left(\mathrm{id} \otimes L_{\prec \mathcal{A}}(x)\right) \Delta_{\prec}(y)+\left(R_{\mathcal{A}}(y) \otimes \mathrm{id}\right) \Delta_{\prec}(y), \\
\Delta_{\succ}\left(x *_{\mathcal{A}} y\right) & =\left(\mathrm{id} \otimes L_{\prec_{\mathcal{A}}}(x)\right) \Delta_{\succ}(y)+\left(R_{\prec_{\mathcal{A}}}(y) \otimes \mathrm{id}\right) \Delta_{\succ}(y), \\
\beta_{\prec}\left(a^{*} *_{\mathcal{A}^{*}} b^{*}\right) & =\left(\operatorname{id} \otimes L_{\prec_{\mathcal{A}^{*}}}\left(a^{*}\right)\right) \beta_{\prec}\left(b^{*}\right)+\left(R_{\mathcal{A}^{*}}\left(b^{*}\right) \otimes \mathrm{id}\right) \beta_{\prec}\left(a^{*}\right)
\end{aligned}
$$




$$
\begin{aligned}
& \beta_{\succ}\left(a^{*} * \mathcal{A}^{*} b^{*}\right)=\left(\operatorname{id} \otimes L_{\mathcal{A}^{*}}\left(a^{*}\right)\right) \beta_{\succ}\left(b^{*}\right)+\left(R_{\prec_{\mathcal{A}^{*}}}\left(b^{*}\right) \otimes \mathrm{id}\right) \beta_{\succ}\left(a^{*}\right), \\
& \left(L_{\mathcal{A}}(x) \otimes \mathrm{id}-\mathrm{id} \otimes R_{\prec_{\mathcal{A}}}(x)\right) \Delta_{\prec}(y)+\sigma\left[\left(L_{\succ \mathcal{A}}(y) \otimes(-\mathrm{id}) \otimes R_{\mathcal{A}}(y)\right) \Delta_{\prec}(y)\right]=0, \\
& \left(L_{\mathcal{A}^{*}}\left(a^{*}\right) \otimes \mathrm{id}-\mathrm{id} \otimes R_{\prec_{\mathcal{A}^{*}}}\left(a^{*}\right)\right) \beta_{\prec}\left(b^{*}\right)+\sigma\left[\left(L_{\succ \mathcal{A}^{*}}\left(b^{*}\right) \otimes(-i d) \otimes R_{\mathcal{A}^{*}}\left(b^{*}\right)\right) \beta_{\succ}\left(a^{*}\right)\right]=0, \\
& \text { hold for any } x, y \in \mathcal{A} \text { and } a^{*}, b^{*} \in \mathcal{A}^{*} \text {, where } L_{\mathcal{A}}=L_{\succ_{\mathcal{A}}}+L_{\prec_{\mathcal{A}}}, R_{\mathcal{A}}=R_{\succ_{\mathcal{A}}}+R_{\prec_{\mathcal{A}}}, L_{\mathcal{A}^{*}}= \\
& L_{\succ \mathcal{A}^{*}}+L_{\prec_{\mathcal{A}^{*}}}, R_{\mathcal{A}^{*}}=R_{\succ \mathcal{A}^{*}}+R_{\prec_{\mathcal{A}^{*}}} .
\end{aligned}
$$

We also denote it by $\left(\mathcal{A}, \mathcal{A}^{*}, \Delta_{\succ}, \Delta_{\prec}, \beta_{\succ}, \beta_{\prec}\right)$ or simply $\left(\mathcal{A}, \mathcal{A}^{*}\right)$.

TheOREM 2.14. Let $\left(A, \prec_{A}, \succ_{A}\right)$ and $\left(A^{*}, \prec_{A^{*}}, \succ_{A^{*}}\right)$ be two dendriform algebras. Let $\left(A, *_{A}\right)$ and $\left(A^{*}, *_{A^{*}}\right)$ be the associated associative algebras, respectively. Then, the following conditions are equivalent:

(1) there is a double construction of the Connes cocycle associated with $\left(A, *_{A}\right)$ and $\left(A^{*}, *^{*}\right)$;

(2) $\left(A, A^{*}\right)$ is a dendriform D-bialgebra.

Corollary 2.15. Let $(\mathcal{A}, \succ, \prec)$ be a dendriform algebra and $r \in \mathcal{A} \otimes \mathcal{A}$. Suppose that $r$ is symmetric and $r$ satisfies the equation

$$
r_{12} * r_{13}=r_{13} \prec r_{23}+r_{23} \succ r_{12} .
$$

Then, the maps $\Delta_{\succ}$ and $\Delta_{\prec}$ are defined, respectively, by

$$
\begin{aligned}
& \Delta_{\succ}(x)=\left(i d \otimes L(x)-R_{\prec}(x) \otimes i d\right) r_{\succ}, \\
& \Delta_{\prec}(x)=\left(i d \otimes L_{\succ}(x)-R(x) \otimes i d\right) r_{\prec}, \forall x \in \mathcal{A},
\end{aligned}
$$

where $r_{\succ}=-r$ and $r_{\prec}=r$ induce a dendriform algebra structure on $\mathcal{A}^{*}$ such that $\left(\mathcal{A}, \mathcal{A}^{*}\right)$ is a dendriform D-bialgebra. Equation (2.23) is called a D-equation in $\mathcal{A}$.

Proposition 2.16. Let $(\mathcal{A}, \succ, \prec)$ be a dendriform algebra and let $r \in \mathcal{A} \otimes \mathcal{A}$ be a symmetric solution of the $D$-equation in $\mathcal{A}$. Then, the corresponding double construction of Connes cocycle associated to $\mathcal{A}$ and $\mathcal{A}^{*}$ is given from the products in $\mathcal{A}$ as follows:

$$
\begin{aligned}
a^{*} \prec b^{*} & =-R_{\succ}^{*}\left(r\left(a^{*}\right)\right) b^{*}+L^{*}\left(r\left(b^{*}\right)\right) a^{*}, \\
a^{*} \succ b^{*} & =R^{*}\left(r\left(a^{*}\right)\right) b^{*}-L_{\prec}^{*}\left(r\left(b^{*}\right)\right) a^{*}, \\
a^{*} * b^{*} & =a^{*} \succ b^{*}+a^{*} \prec b^{*}=R_{\prec}^{*}\left(r\left(a^{*}\right)\right) b^{*}+L_{\succ}^{*}\left(r\left(b^{*}\right)\right) a^{*}, \\
x \succ a^{*} & =x \succ r\left(a^{*}\right)-r\left(R^{*}(x) a^{*}\right)+R^{*}(x) a^{*}, \\
x \prec a^{*} & =x \prec r\left(a^{*}\right)+r\left(R_{\succ}^{*}(x) a^{*}\right)-R_{\succ}^{*}(x) a^{*}, \\
x * a^{*} & =x * r\left(a^{*}\right)-r\left(R_{\prec}^{*}(x) a^{*}\right)+R_{\prec}^{*}(x) a^{*}, \\
a^{*} \succ x & =r\left(a^{*}\right) \succ x+r\left(L_{\prec}^{*}(x) a^{*}\right)-L_{\prec}^{*}(x) a^{*}, \\
a^{*} \prec x & =r\left(a^{*}\right) \prec x-r\left(L^{*}(x) a^{*}\right)+L^{*}(x) a^{*}, \\
a^{*} * x & =r\left(a^{*}\right) * x-r\left(L_{\succ}^{*}(x) a^{*}\right)+L_{\succ}^{*}(x) a^{*}
\end{aligned}
$$

for any $x \in \mathcal{A}, a^{*}, b^{*} \in \mathcal{A}^{*}$.

In the sequel, unless otherwise stated, all the parameters belong to the complex field $\mathbb{C}$.

\section{1-dimensional associative algebras}

In this section, we investigate the solutions of the associative Yang-Baxter equation, dendriform algebras structures and classify the solutions of $D$-equations in the case of 1 -dimensional associative algebras.

3.1. Solutions of the associative Yang-Baxter equation. Let $(\mathcal{A}, \cdot)$ be an associative algebra with a basis $\left\{e_{1}\right\}$ and $r=a_{11} e_{1} \otimes e_{1} \in \mathcal{A} \otimes \mathcal{A}$. Then the AYBE becomes

$$
a_{11}^{2}\left(e_{1} \cdot e_{1} \otimes e_{1} \otimes e_{1}+e_{1} \otimes e_{1} \otimes e_{1} \cdot e_{1}-e_{1} \otimes e_{1} \cdot e_{1} \otimes e_{1}\right)=0 .
$$

Proposition 3.1. There are only two non-isomorphic 1-dimensional associative algebras. The solutions of the corresponding associative Yang-Baxter equation are given in Table 1. 
Table 1: Solutions of the 1-dimensional associative Yang-Baxter equation.

\begin{tabular}{cc}
\hline Associative algebra $\mathcal{A}$ & Solutions of the $A Y B E$ \\
\hline $\mathcal{A}_{1}: e_{1} \cdot e_{1}=0$ & $r=a_{11} e_{1} \otimes e_{1}$ \\
\hline $\mathcal{A}_{2}: e_{1} \cdot e_{1}=e_{1}$ & $r=0$ \\
\hline
\end{tabular}

3.1.1. Antisymmetric solutions and Frobenius algebra structures. Using the Proposition 2.9, we obtain the results presented in Table 2 .

Table 2: Antisymmetric solutions and Frobenius algebra structures of 1-dimensional associative algebras.

\begin{tabular}{ccc}
\hline Associative algebra $\mathcal{A}$ & Antisymmetric solutions & Frobenius algebra structures over $\mathcal{A} \oplus \mathcal{A}^{*}$ \\
\hline $\mathcal{A}_{1}$ & $r=0$ & $e_{1} * e_{1}=e_{1} * e_{1}^{*}=e_{1}^{*} * e_{1}=0$ \\
\hline $\mathcal{A}_{2}$ & $r=0$ & $e_{1} * e_{1}=e_{1} ; e_{1} * e_{1}^{*}=e_{1}^{*} ; e_{1}^{*} * e_{1}=e_{1}^{*}$ \\
\hline
\end{tabular}

\subsection{Dendriform algebra structures and classification of solutions of the $D$-equation.}

Proposition 3.2. The compatible dendriform algebra structures in 1-dimensional associative algebras and related solutions of D-equations are given in Table 3.

Table 3: The 1-dimensional dendriform algebras and classification of solutions of D-equations.

\begin{tabular}{ccc}
\hline $\begin{array}{c}\text { Associative } \\
\text { algebra } \mathcal{A}\end{array}$ & $\begin{array}{c}\text { Dendriform } \\
\text { algebra structures }\end{array}$ & $\begin{array}{c}\text { Solutions of } \\
\text { D-equation }\end{array}$ \\
\hline $\mathcal{A}_{1}: e_{1} \cdot e_{1}=0$ & $D_{1}^{1}: e_{1} \prec e_{1}=e_{1} \succ e_{1}=0$ & $r=a_{11} e_{1} \otimes e_{1}$ \\
\hline $\mathcal{A}_{2}: e_{1} \cdot e_{1}=e_{1}$ & $D_{1}^{2}: e_{1} \succ e_{1}=\lambda e_{1}, e_{1} \prec e_{1}=(1-\lambda) e_{1} ; \lambda=0,1$ & $r=a_{11} e_{1} \otimes e_{1}$ \\
\hline
\end{tabular}

Proof Let us consider the associative algebra $\mathcal{A}_{2}$. We set $e_{1} \succ e_{1}=a e_{1} ; e_{1} \prec e_{1}=b e_{1}$. Since $e_{1} \cdot e_{1}=e_{1}$, then $a+b=1$. Moreover, we have the equations

$$
\begin{aligned}
& \left(e_{1} \prec e_{1}\right) \prec e_{1}=e_{1} \prec\left(e_{1} \cdot e_{1}\right), \\
& \left(e_{1} \succ e_{1}\right) \prec e_{1}=e_{1} \succ\left(e_{1} \prec e_{1}\right), \\
& e_{1} \succ\left(e_{1} \succ e_{1}\right)=\left(e_{1} \cdot e_{1}\right) \succ e_{1}
\end{aligned}
$$

which give $a b=0 ; a b=b a$ and $a(a-1)=0$, respectively. Then, we obtain $a=0$ and $b=1$ or $a=1$ and $b=0$ yielding the compatible dendriform algebra structures on $\mathcal{A}_{2}$. The solutions of D-equations in these dendriform algebras are given by direct computation.

3.2.1. Symmetric solutions and Connes cocycles structures. Using the Proposition 2.16, we obtain the following results in Table 4 , where $D_{i}^{j}, i, j \in \mathbb{N}^{*}$, means the $i-t h$ dendriform class associated with the $j-t h$ class of associative algebra.

Table 4: Symmetric solutions and Connes cocycles of 1-dimensional associative algebras.

Dendriform algebra $\quad$ Symmetric solutions $\quad$ Connes cocycles over $\mathcal{A} \oplus \mathcal{A}^{*}$

\begin{tabular}{ccc}
$D_{1}^{1}$ & $a_{11} e_{1} \otimes e_{1}$ & $e_{1} * e_{1}=e_{1} * e_{1}^{*}=e_{1}^{*} * e_{1}=e_{1}^{*} * e_{1}^{*}=0$ \\
\hline$D_{1}^{2}$ & $a_{11} e_{1} \otimes e_{1}$ & $, e_{1}^{*} * e_{1}^{*}=-a_{11} e_{1}^{*}, e_{1}^{*} * e_{1}=a_{11}(\lambda-1) e_{1}+\lambda e_{1}^{*}$ \\
& $e_{1} * e_{1}=e_{1}, e_{1} * e_{1}^{*}=-a_{11} \lambda e_{1}+(1-\lambda) e_{1}^{*} ; \lambda=0,1$ \\
\hline
\end{tabular}

\section{2-dimensional associative algebras}

Using a similar approach as in the previous section, we now consider 2 -dimensional associative algebras. Let $(\mathcal{A}, \cdot)$ be an associative algebra with a basis $\left\{e_{1}, e_{2}\right\}$ and $r=\sum_{i, j=1}^{2} a_{i j} e_{i} \otimes e_{j} \in \mathcal{A} \otimes \mathcal{A}$. Then, the AYBE (2.8), i.e. $r_{12} r_{13}+r_{13} r_{23}-r_{23} r_{12}=0$, is satisfied for

$$
\begin{aligned}
& r_{12}=\sum_{i, j=1}^{2} a_{i j} e_{i} \otimes e_{j} \otimes 1, \\
& r_{13}=\sum_{i, j=1}^{2} a_{i j} e_{i} \otimes 1 \otimes e_{j}, \\
& r_{23}=\sum_{i, j=1}^{2} a_{i j} 1 \otimes e_{i} \otimes e_{j} .
\end{aligned}
$$


4.1. Solutions of the associative Yang-Baxter equation. The classification of 2-dimensional complex pre-Lie algebras, including the classification of 2-dimensional complex associative algebras, was performed in $[\mathbf{9}$. Then, the 2-dimensional complex associative algebras can be split into 7 classes [4.

Proposition 4.1. The solutions of the associative Yang-Baxter equation (2.8) in 2-dimensional associative algebras are given in Table 5.

Table 5: Solutions of the 2-dimensional associative Yang-Baxter equation.

\begin{tabular}{|c|c|}
\hline Associative algebra $\mathcal{A}$ & Solutions of the $A Y B E$ \\
\hline $\mathcal{A}_{1}: e_{1} \cdot e_{1}=e_{2}$ & $\left(\begin{array}{cc}0 & 0 \\
0 & a_{22}\end{array}\right)$ \\
\hline $\mathcal{A}_{2}: e_{1} \cdot e_{1}=e_{1}, e_{1} \cdot e_{2}=e_{2}$ & $\left.\begin{array}{ll}0 & a_{12} \\
0 & a_{22}\end{array}\right) ;\left(\begin{array}{cc}0 & a_{12} \\
a_{21} & 0\end{array}\right) a_{12}=-a_{21} \neq 0$ \\
\hline $\mathcal{A}_{3}: e_{1} \cdot e_{1}=e_{1}, e_{2} \cdot e_{1}=e_{2}$ & $\left.\begin{array}{cc}0 & 0 \\
a_{21} & a_{22}\end{array}\right) ;\left(\begin{array}{cc}0 & a_{12} \\
a_{21} & 0\end{array}\right) a_{12}=-a_{21} \neq 0$ \\
\hline $\begin{array}{c}\mathcal{A}_{4}: e_{1} \cdot e_{1}=e_{1}, e_{1} \cdot e_{2}=e_{2}, \\
e_{2} \cdot e_{1}=e_{2}\end{array}$ & $\begin{array}{c}\left(\begin{array}{cc}0 & a_{12} \\
0 & 0\end{array}\right) ;\left(\begin{array}{cc}0 & 0 \\
a_{21} & 0\end{array}\right) a_{21} \neq 0 \\
\left(\begin{array}{cc}0 & 0 \\
0 & a_{22}\end{array}\right) a_{22} \neq 0\end{array}$ \\
\hline $\mathcal{A}_{5}: e_{i} \cdot e_{j}=0 ; i, j=1,2$ & $\left(\begin{array}{ll}a_{11} & a_{12} \\
a_{21} & a_{22}\end{array}\right)$ \\
\hline $\mathcal{A}_{6}: e_{2} \cdot e_{2}=e_{2}$ & $\left(\begin{array}{cc}a_{11} & 0 \\
0 & 0\end{array}\right)$ \\
\hline $\mathcal{A}_{7}: e_{1} \cdot e_{1}=e_{1} ; e_{2} \cdot e_{2}=e_{2}$ & $\left.\begin{array}{ll}0 & 0 \\
0 & 0\end{array}\right)$ \\
\hline
\end{tabular}

Proof We set $r=\sum_{i, j} a_{i j} e_{i} \otimes e_{j} \in \mathcal{A} \otimes \mathcal{A}$, where $i, j=1,2$ and $a_{i j} \in \mathbb{C}$. Then, by direct computation of (2.8), we get the results.

4.1.1. Antisymmetric solutions and Frobenius algebra structures. Using the Proposition 2.9. we get the results of Table 6 .

Table 6: Antisymmetric solutions and Frobenius algebra structures of 2-dimensional associative algebras.

\begin{tabular}{ccc}
\hline $\begin{array}{c}\text { Associative } \\
\text { algebra } \mathcal{A}\end{array}$ & $\begin{array}{c}\text { Antisymmetric } \\
\text { solutions }\end{array}$ & $\begin{array}{c}\text { Frobenius algebra } \\
\text { structures over } \mathcal{A} \oplus \mathcal{A}^{*}\end{array}$ \\
\hline $\mathcal{A}_{1}$ & $r=0$ & $e_{1} * e_{1}=e_{2} ; e_{1} * e_{2}^{*}=e_{2}^{*} ; e_{2}^{*} * e_{1}=e_{1}^{*}$ \\
\hline $\mathcal{A}_{2}$ & $a_{12} e_{1} \otimes e_{2}$ & $e_{1} * e_{1}=e_{1} ; e_{1} * e_{2}=e_{2} ; e_{1} * e_{1}^{*}=e_{1}^{*} ; e_{2}^{*} * e_{1}=e_{2}^{*}$ \\
& $+a_{21} e_{2} \otimes e_{1} ;$ & $e_{2}^{*} * e_{2}^{*}=-a_{12} e_{2}^{*} ; e_{2}^{*} * e_{1}^{*}=a_{21} e_{1}^{*} ; e_{1} * e_{2}^{*}=a_{21} e_{1}$ \\
& $a_{12}=-a_{21} \neq 0$ & $e_{2} * e_{2}^{*}=-a_{12} e_{2}+e_{1}^{*} ; e_{2}^{*} * e_{2}=-a_{12} e_{2} ; e_{1}^{*} * e_{1}=a_{21} e_{2}+e_{1}^{*}$ \\
\hline $\mathcal{A}_{3}$ & $a_{12} e_{1} \otimes e_{2}$ & $e_{1} * e_{1}=e_{1} ; e_{2} * e_{1}=e_{2} ; e_{1} * e_{2}^{*}=e_{2}^{*} ; e_{1}^{*} * e_{1}=e_{1}^{*}$ \\
& $+a_{21} e_{2} \otimes e_{1} ;$ & $e_{2}^{*} * e_{2}^{*}=a_{21} e_{2}^{*} ; e_{1}^{*} * e_{2}^{*}=a_{21} e_{1}^{*} ; e_{1} * e_{1}^{*}=-a_{12} e_{2}+e_{1}^{*}$ \\
& $a_{12}=-a_{21} \neq 0$ & $e_{2} * e_{2}^{*}=a_{21} e_{2} ; e_{2}^{*} * e_{1}=-a_{12} e_{1} ; e_{2}^{*} * e_{2}=a_{21} e_{2}+e_{1}^{*}$ \\
\hline $\mathcal{A}_{4}$ & $e_{1} * e_{1}=e_{1} ; e_{1} * e_{2}=e_{2} ; e_{2} * e_{1}=e_{2} ; e_{1}^{*} * e_{1}=e_{1}^{*}$ \\
& $r=0$ & $e_{1} * e_{1}^{*}=e_{1}^{*} e_{2}^{*} * e_{1}=e_{2}^{*} ; e_{2}^{*} * e_{2}=e_{1}^{*}$ \\
\hline $\mathcal{A}_{5}$ & $a_{12} e_{1} \otimes e_{2}$ & $e_{i} * e_{j}=e_{i}^{*} * e_{j}^{*}=e_{i} * e_{j}^{*}=0$ \\
& $+a_{21} e_{2} \otimes e_{1} ;$ & $e_{i}^{*} * e_{j}=0 ; i, j=1,2$ \\
\hline $\mathcal{A}_{6}$ & $r=0$ & \\
\hline $\mathcal{A}_{7}$ & & $e_{21} \neq e_{2}=e_{2} ; e_{2} * e_{2}^{*}=e_{2}^{*} ; e_{2}^{*} * e_{2}=e_{2}^{*}$ \\
& $r=0$ & $e_{1} * e_{1}=e_{1} ; e_{2} * e_{2}=e_{2} ; e_{2} * e_{2}^{*}=e_{2}^{*}$ \\
& & $e_{1}^{*} * e_{1}=e_{1}^{*} ; e_{2}^{*} * e_{2}=e_{2}^{*} ; e_{1} * e_{1}^{*}=e_{1}^{*}$ \\
\hline
\end{tabular}

4.2. Dendriform algebra structures and classification of solutions of the $D$-equation. The classification of 2-dimensional complex dendriform algebras was firstly studied in $2 \mathbf{2 8}$, but 
unfortunately with some mistakes. In fact, there exists a natural anti-isomorphism between dendriform algebras

$$
F\left(x \succ_{1} y\right)=F(y) \prec_{2} F(x), F\left(x \prec_{1} y\right)=F(y) \succ_{2} F(x) .
$$

So, the dendriform algebras appear in terms of pairs. For example, consider the following dendriform algebras given by:

$$
\begin{aligned}
& D_{1}: e_{1} \prec_{1} e_{1}=e_{1}, e_{1} \prec_{1} e_{2}=e_{2} ; \\
& D_{2}: e_{1} \succ_{2} e_{1}=e_{1}, e_{2} \succ_{2} e_{1}=e_{2} ;
\end{aligned}
$$

and the map $F$ defined by $F\left(e_{1}\right)=e_{1}, F\left(e_{2}\right)=e_{2}$. We have $F\left(e_{1} \prec_{1} e_{1}\right)=e_{1}=F\left(e_{1}\right) \succ_{2}$ $F\left(e_{1}\right) ; F\left(e_{1} \prec_{1} e_{2}\right)=e_{2}=F\left(e_{2}\right) \succ_{2} F\left(e_{1}\right)$. Therefore, there exists an anti-isomrphism between the dendriform algebras $D_{1}$ and $D_{2}$. By this property, one can classify dendriform algebras. For that, let $e_{i}, e_{j} \in \mathcal{A} ; i, j=1,2$. Then,

$$
e_{i} \cdot e_{j}=e_{i} \prec e_{j}+e_{i} \succ e_{j}, \text { where } e_{i} \succ e_{j}=\sum_{k=1}^{2} a_{i j}^{k} e_{k}, e_{i} \prec e_{j}=\sum_{k=1}^{2} b_{i j}^{k} e_{k} \text {. }
$$

Computing the equation (4.1) with the condition $e_{i} \cdot e_{j}=\sum_{k=1}^{2}\left(a_{i j}^{k}+b_{i j}^{k}\right) e_{k}$, we get the compatible dendriform algebra structures on the associative algebra. The solutions of $D$-equations in these dendriform algebras are then determined by direct computation. There results the following:

Proposition 4.2. The compatible dendriform algebra structures in 2-dimensional associative algebras and the solutions of related D-equations are given in Table $\%$.

Table 7: 2-dimensional dendriform algebras and classification of solutions of the D-equation.

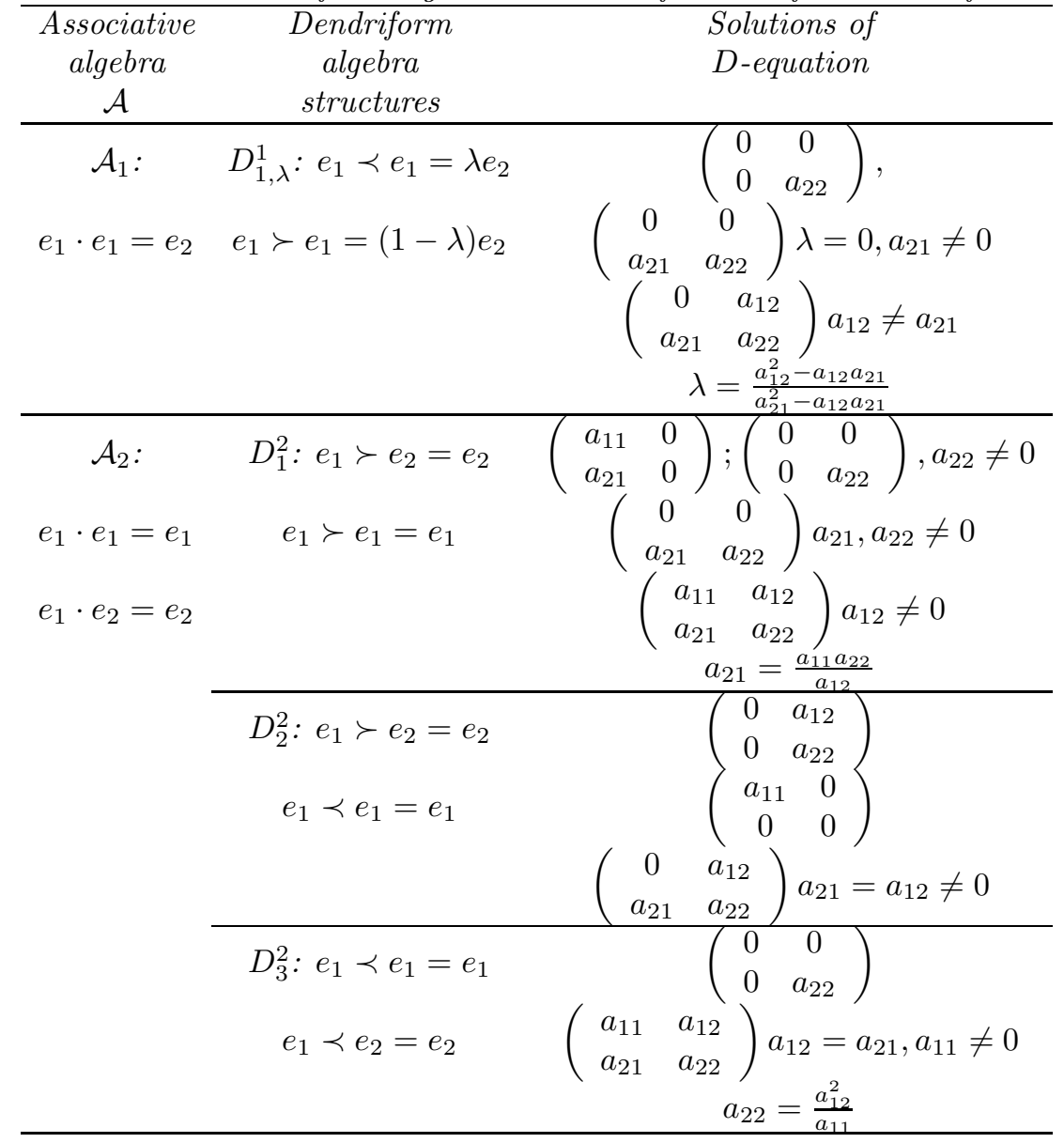




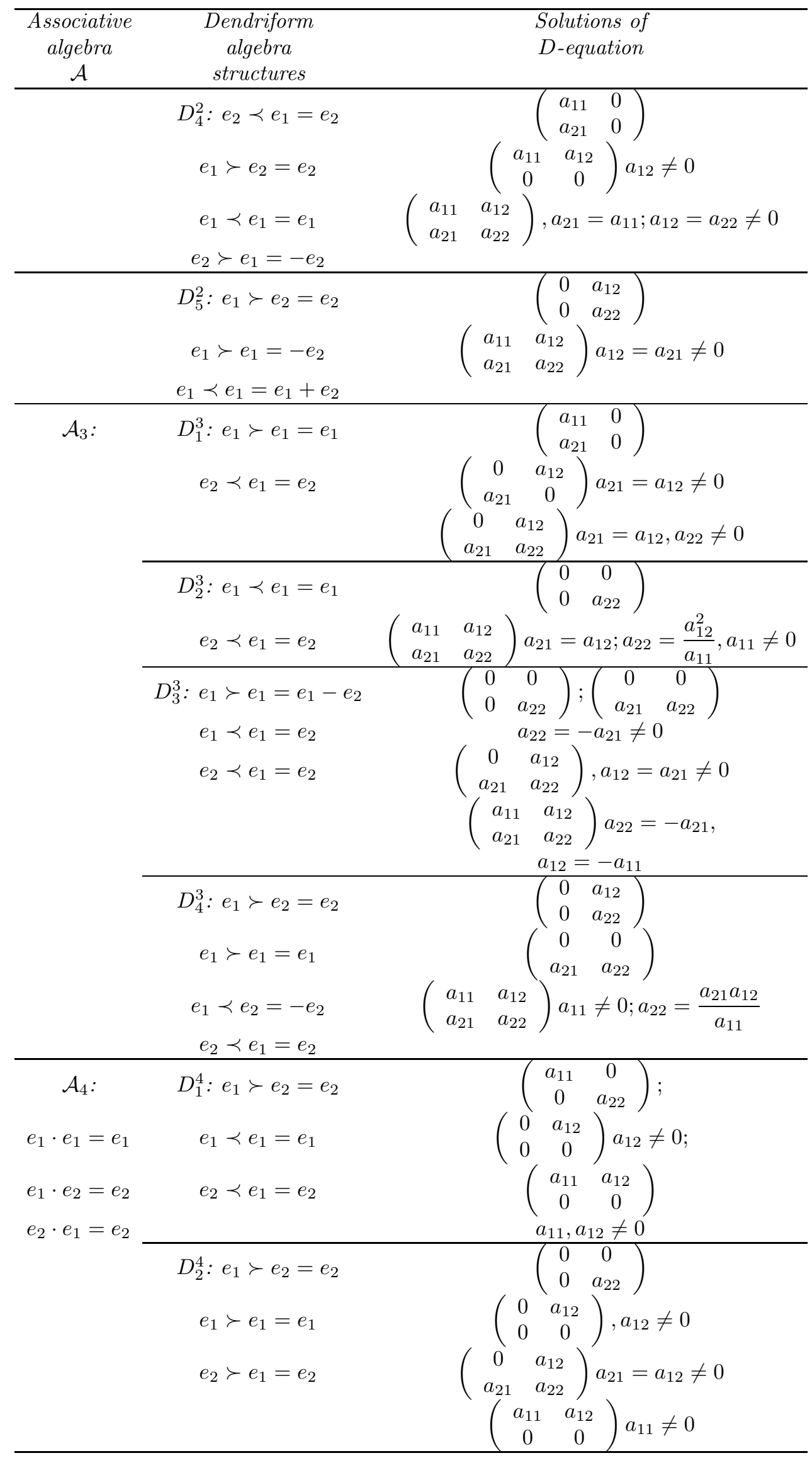




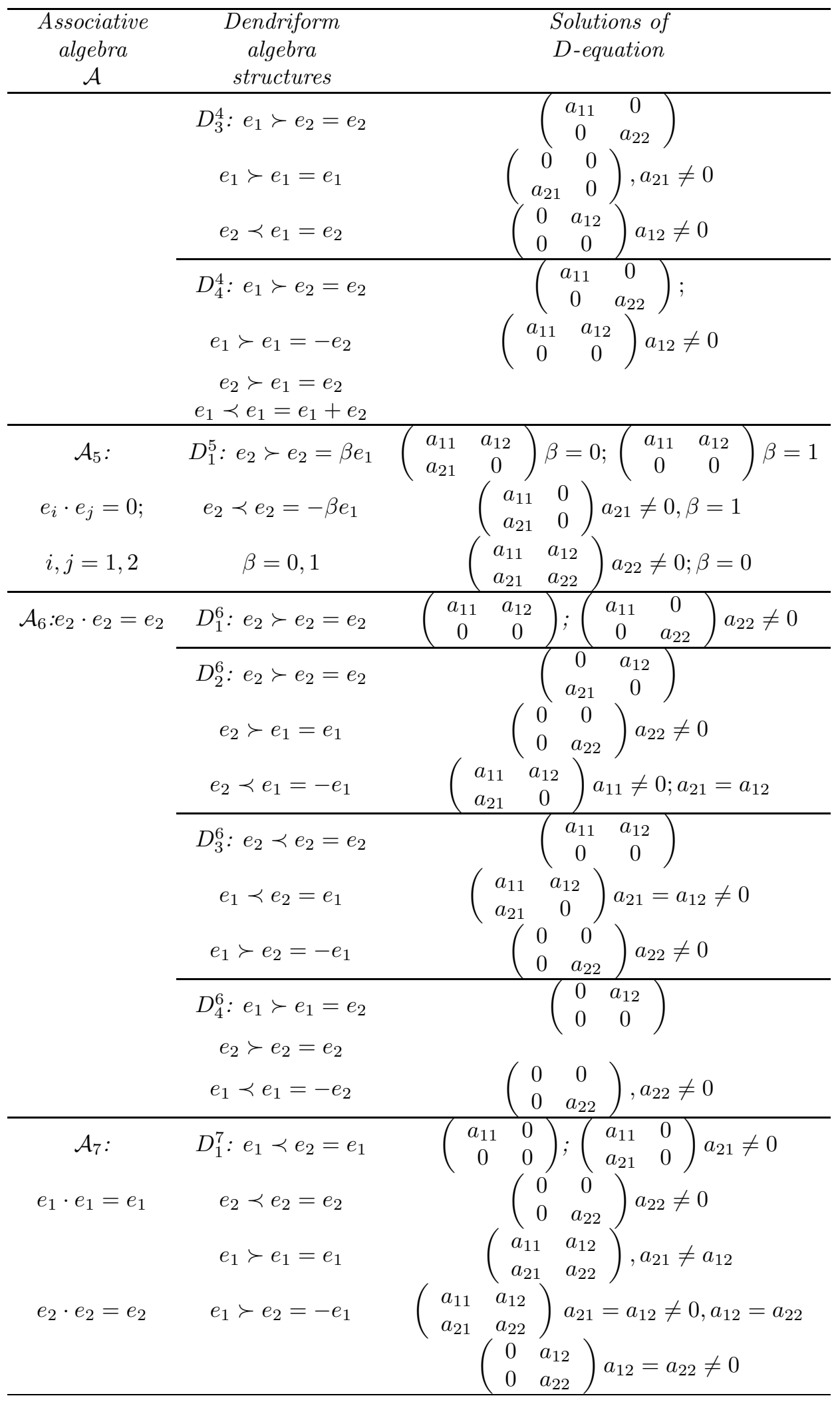




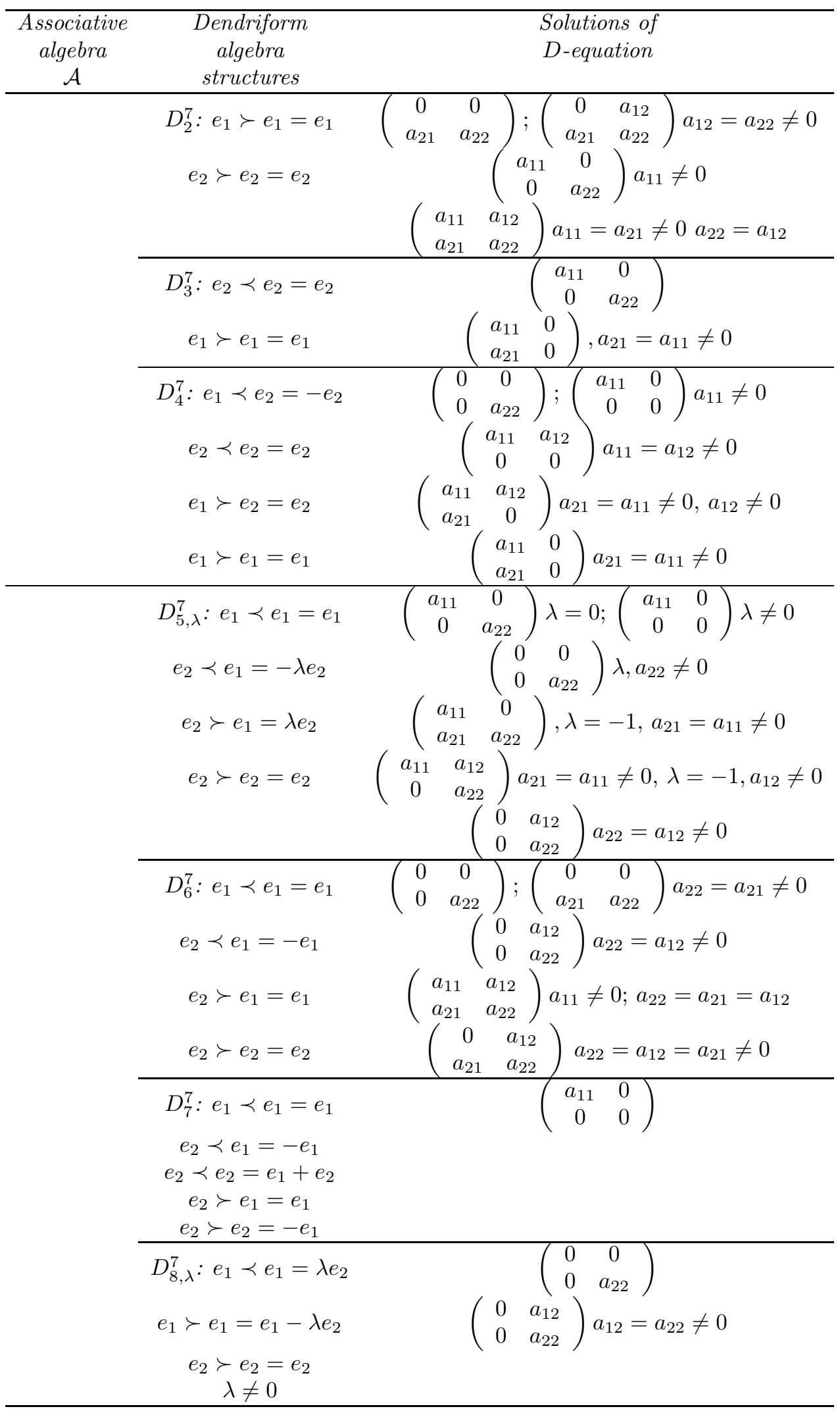




\begin{tabular}{ccc}
\hline $\begin{array}{c}\text { Associative } \\
\text { algebra } \\
\mathcal{A}\end{array}$ & $\begin{array}{c}\text { Dendriform } \\
\text { algebra } \\
\text { structures }\end{array}$ & $\begin{array}{c}\text { Solutions of } \\
\text { D-equation }\end{array}$ \\
\hline$D_{9, \lambda}^{7}: e_{1} \prec e_{1}=\lambda e_{2}$ & $\left(\begin{array}{cc}0 & 0 \\
0 & a_{22}\end{array}\right)$ \\
$e_{2} \prec e_{2}=e_{2}$ & \\
$e_{1} \succ e_{1}=e_{1}-\lambda e_{2}$ & \\
$\lambda \neq 0$ & $\left(\begin{array}{cc}0 & 0 \\
0 & a_{22}\end{array}\right)$ \\
\cline { 2 - 3 }$D_{10, \lambda}^{7}: e_{1} \prec e_{2}=-e_{2}$ & $\left(\begin{array}{c}a_{12} \\
0\end{array}\right) a_{12} \neq 0$ \\
$e_{1} \prec e_{1}=e_{1}-\lambda e_{2}$ & \\
$e_{2} \prec e_{1}=-e_{2}$ & \\
$e_{1} \succ e_{2}=e_{2}$ & \\
$e_{2} \succ e_{1}=e_{2}$ & \\
$e_{1} \succ e_{1}=\lambda e_{2}$ & \\
$e_{2} \succ e_{2}=e_{2}$ & \\
$\lambda \neq 0$ & \\
\hline$D_{11, \lambda}^{7}: e_{1} \prec e_{2}=-e_{2}$ & $\left(\begin{array}{cc}0 \\
0 & a_{22}\end{array}\right)$ \\
$e_{1} \prec e_{1}=e_{1}-\lambda e_{2}$ & $\left(\begin{array}{c}a_{12} \\
0\end{array}\right) a_{12} \neq 0$ \\
$e_{2} \prec e_{1}=-e_{2}$ & \\
$e_{2} \prec e_{2}=e_{2}$ & \\
$e_{1} \succ e_{2}=e_{2}$ & \\
$e_{2} \succ e_{1}=e_{2}$ & \\
$e_{1} \succ e_{1}=\lambda e_{2}$ & \\
$\lambda \neq 0$ & \\
\hline
\end{tabular}

4.2.1. Symmetric solutions and Connes cocycles structures. Using the Proposition 2.16, we obtain the results in Table 8, where $D_{i}^{j}$, with $i, j \in \mathbb{N}^{*}$, means the $i$-th dendriform class associated with the $j-t h$ class of associative algebra.

Table 8: Symmetric solutions and Connes cocycles structures of 2-dimensional associative algebras.

\begin{tabular}{ccc}
\hline $\begin{array}{c}\text { Dendriform } \\
\text { algebra } \\
\text { structures }\end{array}$ & $\begin{array}{c}\text { Symmetric } \\
\text { solutions }\end{array}$ & $\begin{array}{c}\text { Connes cocycles } \\
\text { structures over } \mathcal{A} \oplus \mathcal{A}^{*}\end{array}$ \\
\hline$D_{1, \lambda}^{1}$ & $a_{22} e_{2} \otimes e_{2}$ & $e_{1} * e_{1}=e_{2} ; e_{1} * e_{2}^{*}=\lambda e_{1}^{*} ; e_{2}^{*} * e_{1}=(1-\lambda) e_{1}^{*}$ \\
\hline$D_{1}^{2}$ & $a_{11} e_{1} \otimes e_{1}$ & $e_{1} * e_{1}=e_{1} ; e_{1} * e_{2}=e_{2} ; e_{2}^{*} * e_{1}=e_{2}^{*} ; e_{1}^{*} * e_{2}=-a_{11} e_{2}$ \\
& & $e_{1} * e_{1}^{*}=-a_{11} e_{1} ; e_{2}^{*} * e_{1}^{*}=-a_{11} e_{2}^{*} ; e_{1}^{*} * e_{1}=e_{1}^{*}$ \\
& & $e_{1}^{*} * e_{1}^{*}=-a_{11} e_{1}^{*}$ \\
\cline { 2 - 3 } & $a_{22} e_{2} \otimes e_{2}$ & $e_{1} * e_{1}=e_{1} ; e_{1} * e_{2}=e_{2} ; e_{2}^{*} * e_{1}=e_{2}^{*} ;$ \\
& $a_{22} \neq 0$ & $e_{1} * e_{2}^{*}=-a_{22} e_{2} ; e_{1}^{*} * e_{1}=e_{1}^{*}$ \\
\cline { 2 - 3 } & $a_{11} e_{1} \otimes e_{1}+$ & $e_{1} * e_{1}=e_{1} ; e_{1} * e_{2}=e_{2} ; e_{1}^{*} * e_{1}^{*}=-a_{11} e_{1}^{*}$ \\
& $a_{12} e_{1} \otimes e_{2}+$ & $e_{1} * e_{2}^{*}=-a_{12} e_{1} ; e_{2}^{*} * e_{2}^{*}=a_{22} e_{1}^{*} ; e_{1} * e_{1}^{*}=-a_{11} e_{1}-a_{12} e_{2}$ \\
& $a_{12} e_{2} \otimes e_{1}+$ & $e_{1}^{*} * e_{1}=a_{12} e_{2}+e_{1}^{*} ; e_{2}^{*} * e_{1}=a_{22} e_{2}+e_{2}^{*} ;$ \\
$a_{22} e_{2} \otimes e_{2}$ & $a_{12}^{2}=a_{11} a_{22}$ \\
\hline$D_{2}^{2}$ & $a_{12} e_{1} \otimes e_{2}+$ & $e_{1} * e_{1}=e_{1} ; e_{1} * e_{2}=e_{2} ; e_{1}^{*} * e_{2}^{*}=-a_{12} e_{1}^{*} ; e_{2}^{*} * e_{1}^{*}=-a_{12} e_{1}^{*}$ \\
& $a_{12} e_{2} \otimes e_{1}+$ & $e_{2}^{*} * e_{2}^{*}=-a_{22} e_{1}^{*} ; e_{2}^{*} * e_{2}=-a_{12} e_{2} ; e_{1} * e_{1}^{*}=-a_{12} e_{2}+e_{1}^{*}$ \\
& $a_{22} e_{2} \otimes e_{2}$ & $e_{1} * e_{2}^{*}=-a_{22} e_{2}-a_{12} e_{1} ; e_{2}^{*} * e_{1}=a_{22} e_{2}+e_{2}^{*} ;$ \\
$a_{12} \neq 0$ & \\
\hline
\end{tabular}




\begin{tabular}{|c|c|c|}
\hline $\begin{array}{l}\text { Dendriform } \\
\text { algebra } \\
\text { structures }\end{array}$ & $\begin{array}{l}\text { Symmetric } \\
\text { solutions }\end{array}$ & $\begin{array}{c}\text { Connes cocycles } \\
\text { structures over } \mathcal{A} \oplus \mathcal{A}^{*}\end{array}$ \\
\hline \multirow[t]{2}{*}{$D_{2}^{2}$} & $a_{11} e_{1} \otimes e_{1}$ & $\begin{array}{c}e_{1} * e_{1}=e_{1} ; e_{1} * e_{2}=e_{2} ; e_{2}^{*} * e_{1}=e_{2}^{*} ; e_{1}^{*} * e_{1}^{*}=-a_{11} e_{1}^{*} \\
e_{1}^{*} * e_{1}=-a_{11} e_{1}^{*} ; e_{1} * e_{1}^{*}=e_{1}^{*}\end{array}$ \\
\hline & $a_{22} e_{2} \otimes e_{2}$ & $\begin{array}{c}e_{1} * e_{1}=e_{1} ; e_{1} * e_{2}=e_{2} ; e_{1} * e_{1}^{*}=e_{1}^{*} ; e_{1} * e_{2}^{*}=-a_{22} e_{2} \\
e_{2}^{*} * e_{1}=a_{22} e_{2}+e_{2}^{*}\end{array}$ \\
\hline$D_{3}^{2}$ & $\begin{array}{l}a_{11} e_{1} \otimes e_{1}+ \\
a_{12} e_{1} \otimes e_{2}+ \\
a_{21} e_{2} \otimes e_{1} \\
a_{22} e_{2} \otimes e_{2} \\
a_{21}=a_{12}\end{array}$ & $\begin{array}{c}e_{1} * e_{1}=e_{1} ; e_{1} * e_{2}=e_{2} ; e_{2}^{*} * e_{1}^{*}=a_{12} e_{1}^{*} \\
e_{1}^{*} * e_{2}^{*}=-a_{12} e_{1}^{*} ; e_{1} * e_{1}^{*}=e_{1}^{*} ; e_{1}^{*} * e_{1}^{*}=-a_{11} e_{1}^{*} \\
e_{1}^{*} * e_{1}=-a_{11} e_{1} ; e_{1}^{*} * e_{2}=-a_{11} e_{2}, e_{2}^{*} * e_{1}=-a_{12} e_{1} \\
e_{2}^{*} * e_{2}=-a_{12} e_{2} ; e_{1} * e_{2}^{*}=-a_{22} e_{2}-a_{12} e_{1} \\
e_{2} * e_{2}^{*}=a_{12} e_{2}+a_{11} e_{1}+e_{1}^{*} ; e_{2}^{*} * e_{2}^{*}=-a_{22} e_{1}^{*}\end{array}$ \\
\hline$D_{4}^{2}$ & $a_{11} e_{1} \otimes e_{1}$ & $\begin{array}{c}e_{1} * e_{1}=e_{1} ; e_{1} * e_{2}=e_{2} ; e_{1} * e_{1}^{*}=e_{1}^{*} ; e_{1} * e_{2}^{*}=e_{2}^{*} \\
e_{1}^{*} * e_{1}=-a_{11} e_{1} ; e_{1}^{*} * e_{2}=-a_{11} e_{2} ; e_{2}^{*} * e_{1}=e_{2}^{*} ; e_{1}^{*} * e_{1}^{*}=-a_{11} e_{1}^{*} \\
e_{1}^{*} * e_{2}^{*}=-a_{11} e_{2}^{*} ; e_{2}^{*} * e_{1}^{*}=-a_{11} e_{2}^{*} ; e_{2}^{*} * e_{2}=-a_{11} e_{1}-e_{1}^{*}\end{array}$ \\
\hline \multirow[t]{2}{*}{$\overline{D_{5}^{2}}$} & $\begin{array}{c}a_{11} e_{1} \otimes e_{1}+ \\
a_{12} e_{1} \otimes e_{2}+ \\
a_{12} e_{2} \otimes e_{1} \\
a_{22} e_{2} \otimes e_{2} \\
a_{21}=a_{12} \neq 0\end{array}$ & $\begin{array}{c}e_{1} * e_{1}=e_{1} ; e_{1} * e_{2}=e_{2} ; e_{2}^{*} * e_{2}^{*}=-a_{12} e_{2}^{*} \\
e_{1}^{*} * e_{1}^{*}=-a_{11} e_{1}^{*} ; e_{1} * e_{1}^{*}=e_{1}^{*} ; e_{1}^{*} * e_{1}=-a_{11} e_{1} \\
e_{1}^{*} * e_{2}=-a_{11} e_{2} ; e_{2}^{*} * e_{2}=-a_{12} e_{2} \\
e_{2}^{*} * e_{1}=\left(-a_{12}+a_{22}\right) e_{2}-e_{1}^{*}+e_{2}^{*} \\
e_{1} * e_{2}^{*}=\left(a_{12}-a_{22}\right) e_{2}+e_{1}^{*}+\left(a_{11}-a_{12}\right) e_{1} \\
e_{2}^{*} * e_{1}^{*}=\left(a_{11}-a_{12}\right) e_{1}^{*}-a_{11} e_{2}^{*}\end{array}$ \\
\hline & $a_{22} e_{2} \otimes e_{2}$ & $\begin{array}{c}e_{1} * e_{1}=e_{1} ; e_{1} * e_{2}=e_{2} ; e_{1} * e_{1}^{*}=e_{1}^{*} \\
e_{1}^{*} * e_{1}=e_{1}^{*} ; e_{1} * e_{2}^{*}=-a_{22} e_{2}+e_{1}^{*} \\
e_{2}^{*} * e_{1}=a_{22} e_{2}+e_{2}^{*}-e_{1}^{*}\end{array}$ \\
\hline \multirow[t]{3}{*}{$\overline{D_{1}^{3}}$} & $\begin{array}{l}a_{22} e_{2} \otimes e_{2} \\
a_{22} \neq 0\end{array}$ & $\begin{array}{c}e_{1} * e_{1}=e_{1} ; e_{2} * e_{1}=e_{2} ; e_{1}^{*} * e_{1}=e_{1}^{*} ; e_{2}^{*} * e_{1}=-a_{22} e_{2} \\
e_{1} * e_{2}^{*}=e_{2}^{*}+a_{22} e_{2}\end{array}$ \\
\hline & $a_{11} e_{1} \otimes e_{1}$ & $\begin{array}{c}e_{1} * e_{1}=e_{1} ; e_{2} * e_{1}=e_{2} ; e_{1}^{*} * e_{1}=e_{1}^{*} ; e_{1} * e_{2}^{*}=e_{2}^{*} \\
e_{1} * e_{1}^{*}=-a_{11} e_{1} ; e_{2} * e_{1}^{*}=-a_{11} e_{2} \\
e_{1}^{*} * e_{1}^{*}=-a_{11} e_{1}^{*} ; e_{1}^{*} * e_{2}^{*}=-a_{11} e_{2}^{*}\end{array}$ \\
\hline & $\begin{array}{c}a_{12} e_{1} \otimes e_{2}+ \\
a_{21} e_{2} \otimes e_{1} \\
a_{12}=a_{21} \neq 0\end{array}$ & \\
\hline \multirow[t]{2}{*}{$\overline{D_{2}^{3}}$} & $a_{22} e_{2} \otimes e_{2}$ & $\begin{array}{l}e_{1} * e_{1}=e_{1} ; e_{2} * e_{1}=e_{2} ; e_{1} * e_{1}^{*}=e_{1}^{*} \\
e_{2}^{*} * e_{1}=-a_{22} e_{2} ; e_{1} * e_{2}^{*}=e_{2}^{*}+a_{22} e_{2}\end{array}$ \\
\hline & $a_{11} e_{1} \otimes e_{1}$ & $\begin{array}{c}e_{1} * e_{1}=e_{1} ; e_{2} * e_{1}=e_{2} ; e_{1} * e_{1}^{*}=e_{1}^{*} ; e_{1} * e_{2}^{*}=e_{2}^{*} \\
e_{2} * e_{1}^{*}=-a_{11} e_{2} ; e_{1}^{*} * e_{1}=-a_{11} e_{1}\end{array}$ \\
\hline \multirow[t]{2}{*}{$\overline{D_{3}^{3}}$} & $\begin{array}{l}a_{12} e_{1} \otimes e_{2}+ \\
a_{12} e_{2} \otimes e_{1}+ \\
a_{11} e_{1} \otimes e_{1}+ \\
a_{22} e_{2} \otimes e_{2} \\
a_{22}=a_{11}\end{array}$ & $\begin{array}{c}e_{1} * e_{1}=e_{1} ; e_{2} * e_{1}=e_{2} ; e_{1} * e_{1}^{*}=-a_{11} e_{1} ; e_{2} * e_{1}^{*}=-a_{11} e_{2} \\
e_{2} * e_{2}^{*}=-a_{12} e_{2} ; e_{1}^{*} * e_{1}^{*}=-a_{11} e_{1}^{*} ; e_{2}^{*} * e_{1}^{*}=a_{11} e_{1}^{*} \\
e_{2}^{*} * e_{2}^{*}=-a_{12} e_{2}^{*} ; e_{1} * e_{2}^{*}=a_{11} e_{1}+\left(a_{12}+a_{22}\right) e_{2}+e_{1}^{*}+e_{2}^{*} \\
e_{1}^{*} * e_{1}=a_{11} e_{2}+e_{1}^{*} ; e_{1}^{*} * e_{2}^{*}=\left(-a_{11}-a_{12}\right) e_{1}^{*}-a_{11} e_{2}^{*} \\
e_{2}^{*} * e_{1}=\left(-a_{11}-a_{12}\right) e_{1}+\left(-a_{12}-a_{22}\right) e_{2}-e_{1}^{*} \\
\end{array}$ \\
\hline & $a_{22} e_{2} \otimes e_{2}$ & $\begin{array}{c}e_{1} * e_{1}=e_{1} ; e_{2} * e_{1}=e_{2} ; e_{1} * e_{1}^{*}=e_{1}^{*} ; e_{2} * e_{2}^{*}=-e_{1}^{*} \\
e_{2}^{*} * e_{2}^{*}=a_{22} e_{1}^{*} ; e_{2}^{*} * e_{1}=-e_{1}^{*}+e_{2}^{*} ; e_{1} * e_{2}^{*}=a_{22} e_{2}+e_{1}^{*}+e_{2}^{*}\end{array}$ \\
\hline \multirow[t]{2}{*}{$D_{4}^{3}$} & $a_{22} e_{2} \otimes e_{2}$ & $\begin{array}{c}e_{1} * e_{1}=e_{1} ; e_{2} * e_{1}=e_{2} ; e_{2}^{*} * e_{2}^{*}=a_{22} e_{1}^{*} ; e_{2}^{*} * e_{1}=e_{2}^{*} \\
e_{1}^{*} * e_{1}=e_{1}^{*} ; e_{2} * e_{2}^{*}=-e_{1}^{*} ; e_{1} * e_{2}^{*}=a_{22} e_{2}+e_{2}^{*}\end{array}$ \\
\hline & $\begin{array}{c}a_{11} e_{1} \otimes e_{1}+ \\
a_{12} e_{1} \otimes e_{2}+ \\
a_{12} e_{2} \otimes e_{1}+ \\
a_{22} e_{2} \otimes e_{2} \\
a_{11} a_{22}=a_{12}^{2} ; a_{11} \neq 0\end{array}$ & $\begin{array}{c}e_{1} * e_{1}=e_{1} ; e_{2} * e_{1}=e_{2} ; e_{1}^{*} * e_{1}^{*}=-a_{11} e_{1}^{*} ; e_{2}^{*} * e_{1}^{*}=-a_{11} e_{2}^{*} \\
e_{2}^{*} * e_{2}^{*}=\left(a_{22}-a_{12}\right) e_{1}^{*}-2 a_{12} e_{2}^{*} ; e_{1}^{*} * e_{2}^{*}=-a_{11} e_{2}^{*} \\
e_{2} * e_{1}^{*}=-a_{11} e_{2} ; e_{1} * e_{2}^{*}=-a_{12} e_{1}+\left(a_{12}+a_{22}\right) e_{2}+e_{2}^{*} \\
e_{2} * e_{2}^{*}=-a_{11} e_{1}-2 a_{12} e_{2}-e_{1}^{*} ; e_{1} * e_{1}^{*}=-a_{11} e_{1} \\
e_{2}^{*} * e_{1}=-a_{12} e_{2}+e_{2}^{*} ; e_{1}^{*} * e_{1}=a_{12} e_{1}+e_{1}^{*}\end{array}$ \\
\hline$D_{1}^{4}$ & $\begin{array}{l}a_{11} e_{1} \otimes e_{1}+ \\
a_{22} e_{2} \otimes e_{2}\end{array}$ & $\begin{array}{c}e_{1} * e_{1}=e_{1} ; e_{1} * e_{2}=e_{2} ; e_{2} * e_{1}=e_{2} ; e_{2}^{*} * e_{1}=e_{2}^{*} \\
e_{1}^{*} * e_{1}^{*}=-a_{11} e_{1}^{*} ; e_{1}^{*} * e_{2}^{*}=-a_{11} e_{2}^{*} ; e_{1}^{*} * e_{2}^{*}=-a_{11} e_{2}^{*} \\
e_{1} * e_{2}^{*}=e_{2}^{*} ; e_{2}^{*} * e_{1}^{*}=-a_{11} e_{2}^{*} ; e_{2} * e_{1}^{*}=-a_{11} e_{2} \\
e_{1} * e_{1}^{*}=e_{1}^{*} ; e_{1}^{*} * e_{1}=-a_{11} e_{1}\end{array}$ \\
\hline
\end{tabular}




\begin{tabular}{|c|c|c|}
\hline $\begin{array}{l}\text { Dendriform } \\
\text { algebra } \\
\text { structures }\end{array}$ & $\begin{array}{l}\text { Symmetric } \\
\text { solutions }\end{array}$ & $\begin{array}{l}\text { Connes cocycles } \\
\text { structures over } \mathcal{A} \oplus \mathcal{A}^{*}\end{array}$ \\
\hline \multirow[t]{2}{*}{$D_{2}^{4}$} & $a_{11} e_{1} \otimes e_{1}$ & $\begin{array}{c}e_{1} * e_{1}=e_{1} ; e_{1} * e_{2}=e_{2} ; e_{2}^{*} * e_{2}=a_{11} e_{1}+e_{1}^{*} \\
e_{2}^{*} * e_{1}=e_{2}^{*} ; e_{1} * e_{1}^{*}=-a_{11} e_{1} \\
e_{1}^{*} * e_{1}^{*}=-a_{11} e_{1}^{*} ; e_{2}^{*} * e_{1}^{*}=-a_{11} e_{2}^{*} ; e_{2} * e_{1}=e_{2} \\
e_{2} * e_{1}^{*}=-a_{11} e_{2} ; e_{1}^{*} * e_{2}=-a_{11} e_{2} ; e_{1}^{*} * e_{1}=e_{1}^{*}\end{array}$ \\
\hline & $\begin{array}{l}a_{22} e_{2} \otimes e_{2}+ \\
a_{12} e_{1} \otimes e_{2}+ \\
a_{21} e_{2} \otimes e_{1}\end{array}$ & $\begin{array}{c}e_{1} * e_{1}=e_{1} ; e_{1} * e_{1}^{*}=-a_{12} e_{2} ; e_{2} * e_{2}^{*}=-a_{12} e_{2} \\
e_{2} * e_{1}=e_{2} ; e_{2}^{*} * e_{2}=e_{1}^{*} ; e_{1} * e_{2}^{*}=-a_{12} e_{1}-a_{22} e_{2} \\
e_{2}^{*} * e_{1}=e_{2}^{*}+a_{12} e_{2} ; e_{1}^{*} * e_{1}=e_{1}^{*}-a_{12} e_{2} \\
e_{1} * e_{2}=e_{2} ; e_{1}^{*} * e_{2}^{*}=-a_{12} e_{1}^{*} ; e_{2}^{*} * e_{1}^{*}=-2 a_{12} e_{1}^{*} \\
e_{2}^{*} * e_{2}^{*}=\left(a_{12}-a_{22}\right) e_{1}^{*}-a_{12} e_{2}^{*} \\
\end{array}$ \\
\hline$D_{3}^{4}$ & $\begin{array}{l}a_{11} e_{1} \otimes e_{1}+ \\
a_{22} e_{2} \otimes e_{2}\end{array}$ & $\begin{array}{l}e_{1} * e_{1}=e_{1} ; e_{1} * e_{2}=e_{2} ; e_{1}^{*} * e_{1}^{*}=-a_{11} e_{1}^{*} ; e_{1}^{*} * e_{2}^{*}=-a_{11} e_{2}^{*} \\
e_{2} * e_{1}=e_{2} ; e_{1}^{*} * e_{1}=e_{1}^{*} ; e_{2}^{*} * e_{1}^{*}=-a_{11} e_{2}^{*} ; e_{1} * e_{1}^{*}=-a_{11} e_{1} \\
e_{1} * e_{2}^{*}=e_{2}^{*} ; e_{2}^{*} * e_{1}=e_{2}^{*} ; e_{2} * e_{1}^{*}=-a_{11} e_{2}^{*} ; e_{1}^{*} * e_{2}=-a_{11} e_{2}\end{array}$ \\
\hline$D_{4}^{4}$ & $\begin{array}{l}a_{11} e_{1} \otimes e_{1}+ \\
a_{22} e_{2} \otimes e_{2}\end{array}$ & $\begin{array}{c}e_{1} * e_{1}=e_{1} ; e_{1} * e_{2}=e_{2} ; e_{2} * e_{1}=e_{2} ; e_{1} * e_{1}^{*}=e_{1}^{*} \\
e_{2}^{*} * e_{2}^{*}=-a_{22} e_{1}^{*} ; e_{1}^{*} * e_{2}^{*}=-a_{11} e_{2}^{*} ; e_{1}^{*} * e_{1}^{*}=-a_{11} e_{1}^{*} \\
e_{2} * e_{1}^{*}=-a_{11} e_{2} ; e_{1}^{*} * e_{1}=-a_{11} e_{1} ; e_{1}^{*} * e_{2}=-a_{11} e_{2} \\
e_{2}^{*} * e_{2}^{*}=a_{11} e_{1}^{*}-a_{11} e_{2}^{*} ; e_{2}^{*} * e_{2}^{*}=a_{11} e_{1}-a_{22} e_{2}+e_{1}^{*} \\
e_{2}^{*} * e_{1}=-a_{11} e_{1}^{*}+e_{2}^{*}-e_{1}^{*} ; e_{2}^{*} * e_{2}=a_{11} e_{1}+e_{1}^{*}\end{array}$ \\
\hline \multirow[t]{2}{*}{$\begin{array}{c}D_{1}^{\circ} \\
\beta=0,1\end{array}$} & $\begin{aligned} a_{11} e_{1} & \otimes e_{1}+ \\
a_{12} e_{1} & \otimes e_{2}+ \\
a_{12} e_{21} & \otimes e_{1}+ \\
a_{22} e_{2} & \otimes e_{2}\end{aligned}$ & $\begin{array}{c}e_{i} * e_{j}=e_{i}^{*} * e_{j}=0 \\
e_{i} * e_{j}^{*}=e_{i}^{*} * e_{j}^{*}=0 \\
i, j=1,2 ; \beta=0\end{array}$ \\
\hline & $a_{11} e_{1} \otimes e_{1}$ & $e_{1}^{*} * e_{2}=e_{2}^{*} ; e_{2} * e_{1}^{*}=-e_{2}^{*} ; \beta=1$ \\
\hline$D_{1}^{6}$ & $\begin{array}{c}a_{11} e_{1} \otimes e_{1}+ \\
a_{22} e_{2} \otimes e_{2}\end{array}$ & $\begin{array}{r}e_{2} * e_{2}=e_{2} ; e_{2}^{*} * e_{2}^{*}=-a_{22} e_{2}^{*} \\
e_{2} * e_{2}^{*}=-a_{22} e_{2} ; e_{2}^{*} * e_{2}=e_{2}^{*}\end{array}$ \\
\hline \multirow[t]{2}{*}{$D_{2}^{6}$} & $a_{22} e_{2} \otimes e_{2}$ & $\begin{array}{c}e_{2} * e_{2}=e_{2} ; e_{2}^{*} * e_{2}=e_{2}^{*} ; e_{2}^{*} * e_{2}^{*}=-a_{22} e_{2}^{*} ; e_{1}^{*} * e_{2}=e_{1}^{*} \\
e_{1}^{*} * e_{1}^{*}=a_{22} e_{1}^{*} ; e_{1}^{*} * e_{1}=a_{22} e_{1} ; e_{2}^{*} * e_{1}=-a_{22} e_{1} \\
e_{2}^{*} * e_{2}^{*}=-a_{22} e_{2} ; e_{1} * e_{1}^{*}=-a_{22} e_{2}-e_{2}^{*}\end{array}$ \\
\hline & $\begin{array}{l}a_{11} e_{1} \otimes e_{1}+ \\
a_{12} e_{1} \otimes e_{2}+ \\
a_{12} e_{2} \otimes e_{1}\end{array}$ & $\begin{array}{c}e_{2} * e_{2}=e_{2} ; e_{2}^{*} * e_{2}=e_{2}^{*} ; e_{1}^{*} * e_{2}=e_{1}^{*} ; e_{1} * e_{1}^{*}=-e_{2}^{*} \\
e_{2} * e_{1}^{*}=-a_{12} e_{2} ; e_{1}^{*} * e_{1}=a_{11} e_{1} ; e_{2}^{*} * e_{1}=a_{12} e_{1} \\
e_{1}^{*} * e_{2}^{*}=-a_{12} e_{2}^{*}+a_{12} e_{1}^{*} ; e_{1}^{*} * e_{1}^{*}=a_{11} e_{1}^{*}\end{array}$ \\
\hline \multirow[t]{2}{*}{$D_{3}^{6}$} & $a_{22} e_{2} \otimes e_{2}$ & $\begin{array}{c}e_{2} * e_{2}=e_{2} ; e_{2} * e_{1}^{*}=e_{1}^{*} ; e_{2} * e_{2}^{*}=e_{2}^{*} \\
e_{2}^{*} * e_{1}^{*}=-a_{22} e_{1}^{*} ; e_{1} * e_{1}^{*}=-e_{2}^{*}-a_{22} e_{2} \\
\end{array}$ \\
\hline & $\begin{array}{l}a_{11} e_{1} \otimes e_{1}+ \\
a_{12} e_{1} \otimes e_{2}+ \\
a_{12} e_{2} \otimes e_{1}\end{array}$ & $\begin{array}{c}e_{2} * e_{2}=e_{2} ; e_{2}^{*} * e_{2}^{*}=a_{12} e_{2}^{*} ; e_{2} * e_{1}^{*}=-a_{12} e_{2} \\
e_{2} * e_{1}^{*}=e_{1}^{*}+a_{11} e_{1} ; e_{2} * e_{2}^{*}=e_{2}^{*}+a_{12} e_{2} \\
e_{1} * e_{1}^{*}=-e_{2}^{*}-a_{12} e_{1} ; e_{1}^{*} * e_{1}^{*}=a_{11} e_{2}^{*}-a_{12} e_{1}^{*}\end{array}$ \\
\hline$D_{4}^{6}$ & $a_{22} e_{2} \otimes e_{2}$ & $\begin{array}{c}e_{2} * e_{2}=e_{2} ; e_{2}^{*} * e_{1}=e_{1}^{*} ; e_{2}^{*} * e_{2}^{*}=-a_{22} e_{2}^{*} \\
e_{2} * e_{2}^{*}=-a_{22} e_{2} ; e_{1} * e_{2}^{*}=-e_{1}^{*} ; e_{2}^{*} * e_{2}=e_{2}^{*} \\
\end{array}$ \\
\hline$D_{1}^{7}$ & $\begin{array}{l}a_{11} e_{1} \otimes e_{1}+ \\
a_{12} e_{1} \otimes e_{2}+ \\
a_{21} e_{2} \otimes e_{1} \\
a_{22} e_{2} \otimes e_{2} \\
a_{12}=a_{21}\end{array}$ & $\begin{array}{c}e_{1} * e_{1}=e_{1} ; e_{2} * e_{2}=e_{2} ; e_{2}^{*} * e_{1}^{*}=-a_{12} e_{2}^{*} ; e_{2}^{*} * e_{2}^{*}=-a_{22} e_{2}^{*} \\
e_{1} * e_{2}^{*}=-a_{11} e_{1} ; e_{1} * e_{2}^{*}=-a_{12} e_{1} ; e_{2} * e_{1}^{*}=-a_{12} e_{2}^{*} \\
e_{2} * e_{2}^{*}=-a_{22} e_{2} ; e_{1}^{*} * e_{2}=-a_{12} e_{2} ; e_{2}^{*} * e_{1}=-a_{12} e_{1} \\
e_{1}^{*} * e_{1}^{*}=-a_{11} e_{1}^{*} ; e_{1}^{*} * e_{2}^{*}=-a_{12} e_{1}^{*} \\
e_{1}^{*} * e_{1}=a_{12} e_{2}+e_{1}^{*} ; e_{2}^{*} * e_{2}=a_{12} e_{1}+e_{2}^{*} \\
\end{array}$ \\
\hline$D_{2}^{7}$ & $\begin{array}{l}a_{11} e_{1} \otimes e_{1}+ \\
a_{12} e_{1} \otimes e_{2}+ \\
a_{21} e_{2} \otimes e_{1} \\
a_{22} e_{2} \otimes e_{2} \\
a_{12}=a_{21}\end{array}$ & $\begin{aligned} e_{1} * e_{1}= & e_{1} ; e_{2} * e_{2}=e_{2} ; e_{1} * e_{1}^{*}=-a_{11} e_{1} ; e_{1} * e_{2}^{*}=-a_{12} e_{1} \\
e_{1}^{*} * e_{2}= & -a_{12} e_{2} ; e_{2}^{*} * e_{2}=-a_{22} e_{2} ; e_{1}^{*} * e_{1}^{*}=\left(-a_{11}-a_{12}\right) e_{1}^{*} \\
e_{2}^{*} * e_{1}^{*}= & -a_{22} e_{1}^{*} ; e_{2}^{*} * e_{2}^{*}=-a_{22} e_{2}^{*} ; e_{1}^{*} * e_{2}^{*}=-a_{12} e_{2}^{*}-a_{12} e_{1}^{*} \\
& e_{2} * e_{1}^{*}=-a_{12} e_{2}+e_{1}^{*} ; e_{2} * e_{2}^{*}=a_{12} e_{1}+e_{2}^{*} \\
& e_{1}^{*} * e_{1}=-a_{12} e_{1}+\left(a_{12}-a_{22}\right) e_{2}+e_{1}^{*}-e_{2}^{*}\end{aligned}$ \\
\hline$D_{3}^{7}$ & $\begin{array}{c}a_{11} e_{1} \otimes e_{1}+ \\
a_{22} e_{2} \otimes e_{2}\end{array}$ & $\begin{array}{l}e_{1} * e_{1}=e_{1} ; e_{2} * e_{2}=e_{2} ; e_{1}^{*} * e_{1}^{*}=-a_{11} e_{1}^{*} ; e_{2}^{*} * e_{2}^{*}=-a_{22} e_{2}^{*} \\
e_{1} * e_{1}^{*}=-a_{11} e_{1} ; e_{2} * e_{2}^{*}=e_{2}^{*} ; e_{1}^{*} * e_{1}=e_{1}^{*} ; e_{2}^{*} * e_{2}=-a_{22} e_{2}\end{array}$ \\
\hline
\end{tabular}




\begin{tabular}{|c|c|c|}
\hline $\begin{array}{l}\text { Dendriform } \\
\text { algebra } \\
\text { structures }\end{array}$ & $\begin{array}{l}\text { Symmetric } \\
\text { solutions }\end{array}$ & $\begin{array}{l}\text { Connes cocycles } \\
\text { structures over } \mathcal{A} \oplus \mathcal{A}^{*}\end{array}$ \\
\hline$D_{4}^{7}$ & $\begin{array}{l}a_{11} e_{1} \otimes e_{1}+ \\
a_{12} e_{1} \otimes e_{2} \\
a_{21} e_{2} \otimes e_{1} \\
a_{21}=a_{12}\end{array}$ & $\begin{array}{c}e_{1} * e_{1}=e_{1} ; e_{2} * e_{2}=e_{2} ; e_{2}^{*} * e_{1}^{*}=-a_{11} e_{2}^{*} ; e_{1}^{*} * e_{2}^{*}=-a_{12} e_{2}^{*} \\
e_{1} * e_{1}^{*}=-a_{11} e_{1} ; e_{1} * e_{2}^{*}=-a_{12} e_{1} ; e_{2} * e_{1}^{*}=-a_{12} e_{1}^{*} \\
e_{1}^{*} * e_{2}=-a_{12} e_{2} ; e_{2}^{*} * e_{1}=e_{2}^{*} ; e_{1}^{*} * e_{1}^{*}=-a_{11} e_{1}^{*} \\
e_{2}^{*} * e_{2}^{*}=-a_{12} e_{2}^{*} ; e_{1}^{*} * e_{1}=a_{12} e_{2}+e_{1}^{*} \\
e_{2} * e_{2}^{*}=\left(-a_{11}+a_{12}\right) e_{1}-a_{12} e_{2}-e_{1}^{*}+e_{2}^{*}\end{array}$ \\
\hline \multirow[t]{3}{*}{$D_{5, \lambda}^{7}$} & $a_{11} e_{1} \otimes e_{1}$ & $\begin{array}{c}e_{1} * e_{1}=e_{1} ; e_{2} * e_{2}=e_{2} ; e_{1} * e_{1}^{*}=e_{1}^{*} ; e_{1}^{*} * e_{1}=-a_{11} e_{1} \\
e_{1} * e_{2}^{*}=-\lambda e_{2}^{*} ; e_{1}^{*} * e_{1}^{*}=-a_{11} e_{1}^{*} ; e_{1}^{*} * e_{2}^{*}=a_{11} \lambda e_{2}^{*} \\
e_{2}^{*} * e_{2}=a_{11} \lambda e_{1}+\lambda e_{1}^{*}+e_{2}^{*}\end{array}$ \\
\hline & $a_{22} e_{2} \otimes e_{2}$ & $\begin{array}{c}e_{1} * e_{1}=e_{1} ; e_{2} * e_{2}=e_{2} ; e_{1} * e_{1}^{*}=e_{1}^{*} ; e_{1} * e_{2}^{*}=-a_{22} \lambda e_{2} \\
e_{2} * e_{2}^{*}=-a_{22} e_{2} ; e_{1} * e_{2}^{*}=-\lambda e_{2}^{*} ; e_{2}^{*} * e_{2}=\lambda e_{1}^{*}+e_{2}^{*} \\
e_{2}^{*} * e_{2}^{*}=-\lambda a_{22} e_{1}^{*}-a_{22} e_{2}^{*}\end{array}$ \\
\hline & $\begin{array}{l}a_{11} e_{1} \otimes e_{1} \\
a_{12} e_{1} \otimes e_{2} \\
a_{21} e_{2} \otimes e_{1} \\
a_{22} e_{2} \otimes e_{2} \\
a_{21}=a_{12}\end{array}$ & $\begin{array}{c}e_{1} * e_{1}=e_{1} ; e_{2} * e_{2}=e_{2} ; e_{1} * e_{1}^{*}=e_{1}^{*} ; e_{2} * e_{1}^{*}=-a_{12} e_{2} \\
e_{2} * e_{2}^{*}=-a_{22} e_{2} ; e_{1}^{*} * e_{1}=-a_{11} e_{1} ; e_{1}^{*} * e_{2}=-a_{12} e_{2} \\
e_{2}^{*} * e_{1}=-a_{12} e_{1} ; e_{1}^{*} * e_{2}^{*}=-a_{12} e_{2}^{*} ; e_{1}^{*} * e_{1}^{*}=-a_{11} e_{1}^{*} \\
e_{2}^{*} * e_{1}^{*}=-a_{12} e_{2}^{*} ; e_{2}^{*} * e_{2}=-a_{12} e_{2}-e_{1}^{*}+e_{2}^{*} \\
e_{2}^{*} * e_{2}^{*}=-a_{22} e_{1}^{*}+\left(-a_{22}-a_{12}\right) e_{2}^{*}\end{array}$ \\
\hline$D_{6}^{7}$ & $\begin{array}{c}a_{11} e_{1} \otimes e_{1} \\
a_{12} e_{1} \otimes e_{2} \\
a_{21} e_{2} \otimes e_{1} \\
a_{22} e_{2} \otimes e_{2} \\
a_{21}=a_{12}=a_{22}\end{array}$ & $\begin{array}{c}e_{1} * e_{1}=e_{1} ; e_{2} * e_{2}=e_{2} ; e_{1} * e_{1}^{*}=-a_{12} e_{1}+e_{1}^{*}-e_{2}^{*} \\
e_{1} * e_{2}^{*}=-a_{12} e_{1} ; e_{2} * e_{1}^{*}=-a_{12} e_{2} ; e_{2} * e_{2}^{*}=-a_{22} e_{2} \\
e_{1}^{*} * e_{2}^{*}=-a_{12} e_{1}^{*} ; e_{2}^{*} * e_{1}=-a_{12} e_{1} ; e_{1}^{*} * e_{1}=-a_{11} e_{1} \\
e_{2}^{*} * e_{2}^{*}=\left(-a_{22}-a_{12}\right) e_{2}^{*} ; e_{1}^{*} * e_{2}=a_{11} e_{1}+a_{12} e_{2}+e_{1}^{*} \\
e_{2}^{*} * e_{1}^{*}=-a_{12} e_{1}^{*}+a_{12} e_{2}^{*} ; e_{2}^{*} * e_{2}=a_{12} e_{1}-a_{12} e_{2}+e_{2}^{*} \\
e_{1}^{*} * e_{1}^{*}=\left(-a_{11}-a_{12}\right) e_{1}^{*}+a_{11} e_{2}^{*}\end{array}$ \\
\hline$D_{7}^{7}$ & $a_{11} e_{1} \otimes e_{1}$ & $\begin{array}{c}e_{1} * e_{1}=e_{1} ; e_{2} * e_{2}=e_{2} ; e_{2} * e_{1}^{*}=e_{2}^{*} ; e_{2} * e_{2}^{*}=e_{2}^{*} \\
e_{1}^{*} * e_{1}=-a_{11} e_{1} ; e_{1}^{*} * e_{2}=a_{11} e_{1}+e_{1}^{*}-e_{2}^{*} \\
e_{1} * e_{1}^{*}=e_{1}^{*}-e_{2}^{*} ; e_{1}^{*} * e_{1}^{*}=-a_{11} e_{1}^{*}+a_{11} e_{2}^{*}\end{array}$ \\
\hline$D_{8, \lambda}^{7}$ & $a_{22} e_{2} \otimes e_{2}$ & $\begin{array}{c}e_{1} * e_{1}=e_{1} ; e_{2} * e_{2}=e_{2} ; e_{1} * e_{2}^{*}=\lambda e_{1}^{*} ; e_{2} * e_{2}^{*}=-a_{22} e_{2} \\
e_{1}^{*} * e_{1}=e_{1}^{*} ; e_{2}^{*} * e_{1}=-\lambda e_{1}^{*} ; e_{2}^{*} * e_{2}=e_{2}^{*} ; e_{2}^{*} * e_{2}^{*}=-a_{22} e_{2}^{*}\end{array}$ \\
\hline$D_{9, \lambda}^{7}$ & $a_{22} e_{2} \otimes e_{2}$ & $\begin{array}{c}e_{1} * e_{1}=e_{1} ; e_{2} * e_{2}=e_{2} ; e_{1} * e_{2}^{*}=\lambda e_{1}^{*} ; e_{2}^{*} * e_{2}=-a_{22} e_{2} \\
e_{2} * e_{2}^{*}=e_{2}^{*} ; e_{1}^{*} * e_{1}=e_{1}^{*} ; e_{2}^{*} * e_{1}=-\lambda e_{1}^{*} ; e_{2}^{*} * e_{2}^{*}=-a_{22} e_{2}^{*}\end{array}$ \\
\hline$D_{10, \lambda}^{7}$ & $a_{22} e_{2} \otimes e_{2}$ & $\begin{array}{c}e_{1} * e_{1}=e_{1} ; e_{2} * e_{2}=e_{2} ; e_{1} * e_{2}^{*}=-a_{22} e_{2}-\lambda e_{1}^{*}-e_{2}^{*} \\
e_{1} * e_{1}^{*}=e_{1}^{*} ; e_{2}^{*} * e_{2}^{*}=-a_{22} e_{2}^{*} ; e_{2} * e_{2}^{*}=-a_{22} e_{2}-e_{1}^{*} \\
e_{2}^{*} * e_{2}=e_{1}^{*}+e_{2}^{*} ; e_{2}^{*} * e_{1}=\lambda e_{1}^{*}+e_{2}^{*}\end{array}$ \\
\hline$\overline{D_{11, \lambda}^{7}}$ & $a_{22} e_{2} \otimes e_{2}$ & $\begin{array}{c}e_{1} * e_{1}=e_{1} ; e_{2} * e_{2}=e_{2} ; e_{1} * e_{2}^{*}=-a_{22} e_{2}-\lambda e_{1}^{*}-e_{2}^{*} \\
e_{1} * e_{1}^{*}=e_{1}^{*} ; e_{2}^{*} * e_{2}^{*}=-a_{22} e_{2}^{*} ; e_{2} * e_{2}^{*}=e_{2}^{*}-e_{1}^{*} \\
e_{2}^{*} * e_{2}=-a_{22} e_{2}+e_{1}^{*} ; e_{2}^{*} * e_{1}=a_{22} e_{2}+\lambda e_{1}^{*}+e_{2}^{*}\end{array}$ \\
\hline
\end{tabular}

\section{Concluding remarks}

In this work, we gave an overview of the main concepts, definitions and known fundamental results related to the notions of Frobenius algebras, bialgebras, and Connes cocycles. We classified the solutions of the associative Yang-Baxter equation on complex associative algebras in dimensions 1 and 2. In dimension 1, we obtained 2 classes against 7 in dimension 2 . The skewsymmetric solutions enabled us to carry out double constructions of the Frobenius algebras of these associative algebras. Finally, we obtained all compatible dendriform algebras in dimensions 1 and 2, gave a classification of solutions of D-equations and the skew-symmetric Connes cocycles associated with symmetric solutions.

\section{Aknowledgement}

MNH is grateful to Prof C. Bai from Chern Institute of Mathematics, China, for useful discussions, inputs and provided references. This work is partially supported by the Abdus Salam 
International Centre for Theoretical Physics (ICTP, Trieste, Italy) through the Office of External Activities (OEA) - Prj-15. The ICMPA is also in partnership with the Daniel Iagolnitzer Foundation (DIF), France.

\section{References}

[1] M. Aguiar, Infinitesimal Hopf algebras. In New trends in Hopf algebra theory(La Falda, 1999), Contemp. Math. Amer. Math. Soc., Providence, RI, 267 (2000), pp. 1-29.

[2] M. Aguiar, Pre-Poisson algebras. Lett. Math. Phys. 54 (2000), pp. 263-277.

[3] M. Aguiar, On the associative analog of Lie bialgebras. J. Algebra 244 (2001), pp. 492-532.

[4] H. An and C. Bai, From Rota-Baxter algebras to pre-Lie algebras. J. Phys. A: Math. Theor. 41 (2008)015201(19pp).

[5] C. Bai, Double constructions of Frobenius algebras, Connes cocycle and their duality. J. Noncommut. Geom. 4 (2010), pp. 475 - 530.

[6] M. Bordemann, Nondegenerate invariant bilinear forms on non associative algebras. Acta Math. Univ. Comenian. (N.S.) 66 (1997), pp 151-201.

[7] M. Bordemann, T. Filk, C. Nowak, Algebraic classification of actions invariant under generalized flip moves of 2-dimensional graph. J. Math. Phys. 35 (1994), pp. 4964-4988.

[8] R. Brauer, C. Nesbitt, On the regular representations of algebras. Proc. Nat. Acad. Sci. U.S.A. 66 (1937), pp. 236-240.

[9] D. Burde, Simple left-symmetric algebras with solvable Lie algebra. Manuscipta Math. 95, 3 (1998), pp. 397-411.

[10] F. Chapoton, Un théorème de Cartier - Milnor - Moore - Quillen pour les bigèbres dendriformes et les algèbres braces. J. Pure and Appl. Alg. 168 (2002), pp. 1 - 18.

[11] A. Connes Non-commutative differential geometry. Inst. Hautes Etudes Sci. Publ. Math. 62 (1985), pp. 257-360.

[12] K. Ebrahimi-Fard, D. Manchon, F. Patras, New identities in dendriform algebras. J. Algebra 320 (2008), pp. 167-194.

[13] L. Foissy, Les algèbres de Hopf des arbres enracinés décorés II. Bull. Sci. Math. 126 (2002), pp. 249-288.

[14] A. Frabetti, Dialgebra homology of associative algebras. C. R. Acad. Sci. Paris 325 (1997), pp. 135-140.

[15] A. Frabetti, Leibniz homology of dialgebras of matrices. J. Pure. Appl. Alg. 129 (1998), pp. 123-141.

[16] R. Holtkamp, Comparison of Hopf algebras on trees. Arch. Math. (Basel). 80 (2003), pp. 368-383.

[17] R. Holtkamp, On Hopf algebra structures over operad. Adv. Math. 207 (2006), pp. 544-565.

[18] S. A. Joni, G. C. Rota, Coalgebras and bialgebras in combinatories. Stud. Appl. Math. 61 (1979), pp. 93-139.

[19] J. Kock, Frobenius algebras and $2 D$ topological quantum field theories. London Mathematical Society Student Texts, 59. Cambrige University Press, Cambrige, 2004.

[20] J.-L. Loday, Dialgebras, in Dialgebras and related operads. Lecture Notes in Math. 1763 (2002), pp. 7-66.

[21] J.-L. Loday, Arithmetree. J. Algebra 258 (2002), pp. 275-309.

[22] J.-L. Loday, Scindement d'associativité et algèbres de Hopf. Proceedings of the Conference in hounor of Jean Leray, Nantes (2002), Séminaire et Congrès(SMF) 9 (2004), pp. 155-172.

[23] J.-L. Loday, M. Ronco, Hopf algebra of the planar binary trees. Adv. Math. 139 (1998), pp. 293-309.

[24] J.-L. Loday, M. Ronco, Algèbre de Hopf colibres. C.R. Acad. Sci. Paris 337 (2003), pp. 153-158.

[25] M. Ronco, Eulerian idempotents and Milnor-Moore theorem for certain non-cocommutative Hopf algebras. J. Algebra 254 (2002), pp. 151-172.

[26] R. D. Schafer, An introduction to nonassociative algebras. Corrected reprint of the 1966 original, Dover Publications, New York (1995).

[27] K. Yamagata, Frobenius algebras. In Handbook of algebra, Vol. 1, North-Holland, Amsterdam (1996), pp. 841-887.

[28] L. Zhang, The classification of 2-dimensional dendriform algebras. Thesis for Bachloar Degree, Nankai University, (2008).

[29] V. N. Zhelyabin, Jordan bialgebras and their connection with Lie bialgebras. Algebra i Logika 36 (1997), pp. 3-25; English transl. Algebra Logic 36 (1997), pp. 1-16.

(*) University of Abomey-Calavi, International Chair in Mathematical Physics and Applications, ICMPA-UNESCO Chair, 072 BP 50, Cotonou, ReP. of Benin

E-mail address: norbert.hounkonnou@cipma.uac.bj, with copy to hounkonnou@yahoo.fr

$(\dagger)$ University of Abomey-Calavi, International Chair in Mathematical Physics and Applications, ICMPA-UNESCO Chair, 072 BP 50, Cotonou, ReP. of Benin

E-mail address: houndedjid@gmail.com 